\title{
Gilbert damping in binary magnetic multilayers
}

\author{
E. Barati* and M. Cinal \\ Institute of Physical Chemistry, Polish Academy of Sciences, 01-224 Warsaw, Poland
}

(Received 25 June 2016; published 25 April 2017)

\begin{abstract}
We present quantum mechanical calculations of the Gilbert damping constant $\alpha$ in ultrathin $\mathrm{L} 1_{0}[\mathrm{Co} / \mathrm{NM}]_{N}$ superlattices and $(001) \mathrm{fcc}[\mathrm{Co} / \mathrm{NM}]_{N}$ magnetic multilayers built of cobalt and nonmagnetic metals $\mathrm{NM}=\mathrm{Cu}$, $\mathrm{Ag}, \mathrm{Pd}, \mathrm{Pt}$, and $\mathrm{Au}$. The calculations are performed within a realistic nine-orbital tight-binding model of the band structure including spin-orbit interaction. The dependence of $\alpha$ on the stacking number $N$, ferromagnetic and nonmagnetic layer thicknesses as well as the electron scattering rate is investigated. The damping constant is shown to be the sum of a constant term (bulklike) and a $1 / N$ term (due to external surfaces) which arise from interand intraband electronic transitions, respectively. The calculated $\alpha$ is found to be enhanced in the considered multilayers in comparison with its values for bulk Co and their bilayer counterparts with the same total Co thickness. The origin of this enhancement and the variation of $\alpha$ with the geometric structure of the multilayers are further investigated by analyzing the damping contributions from individual atomic layers. The obtained theoretical results for the damping constant are shown to be in good agreement with previous experimental observations in magnetic multilayers. In particular, the experimentally observed linear dependence on the ratio of $\mathrm{NM}(\mathrm{Pd}$ or $\mathrm{Pt})$ and Co layer thicknesses is reproduced in the present calculations.
\end{abstract}

DOI: 10.1103/PhysRevB.95.134440

\section{INTRODUCTION}

A periodic configuration of alternating layers of two or more different materials is called a binary superlattice. Superlattices possess new properties that the constituents do not and therefore they can be utilized in various novel applications. In particular, multiple interfaces present in such systems act as barriers to dislocations and thus greatly improve their mechanical properties, like hardness and shear strength, making them very suitable materials for superhard coatings [1]. In semiconductor superlattices, the presence of quantum wells and the resulting electronic minibands make it possible to tune charge transport and optical properties, which has led to their vast practical applications in optics and electronics [2-4].

Other systems with a similar structure and a great potential for applications are magnetic binary superlattices built of repeated ultrathin ferromagnet/nonmagnet (FM/NM) metallic bilayers. Such multilayers can exhibit oscillatory interlayer exchange coupling [5-7,10-12] and giant magnetorestistance $[5,10,13,14]$ as well as large perpendicular magnetic anisotropy (PMA) due to the magnetocrystalline anisotropy terms arising from the multiple FM/NM interfaces. In particular, large PMA is found in $\mathrm{Fe} / \mathrm{Pt}, \mathrm{Co} / \mathrm{Pd}$, and $\mathrm{Co} / \mathrm{Pt}$ multilayers [15-23], including $\mathrm{L}_{0}$ superlattices [24-27]. Magnetic multilayers with out-of-plane magnetization are essential ingredients in spintronic applications, in particular, they turn out to be suitable in spin-transfer torque devices like magnetic random access memory (MRAM) [28-31]. They have also been employed in domain wall structures with narrow domain walls which are currently a subject of great interest [32-35].

The dynamics of magnetization in metallic systems is strongly affected by spin relaxation processes, in particular the Gilbert damping [36,37], which enters the

\footnotetext{
*Present address: Faculty of Science and Technology and MESA ${ }^{+}$ Institute for Nanotechnology, University of Twente, P.O. Box 217, 7500 AE Enschede, The Netherlands; e.barati@utwente.nl
}

Landau-Lifshitz-Gilbert equation and originates from the spinorbit (SO) coupling in the torque correlation model developed by Kamberský [38]. The Gilbert damping constant $\alpha$ can be enhanced by transfer of spin angular momentum from the ferromagnetic part of the system to its nonmagnetic or antiferromagnetic parts, as described by the spin pumping theory [39]. Another significant source of spin relaxation is the two-magnon scattering [40,41] due to structure imperfections, like surface roughness and disorder at interfaces. Determination of $\alpha$ in magnetic materials is of great interest due to its subtle role in spintronic devices, like magnetic sensors with the demand for large magnetic damping or MRAMs where low damping is desirable. Therefore, magnetic multilayers have been investigated experimentally also because they allow for engineering magnetic damping [25,42-45].

Measurements of the damping constant $\alpha$ in $[\mathrm{FM} / \mathrm{NM}]_{N}$ binary superlattices have been reported by different experimental groups [22,23,25-27,42-52]. The presence of NM layers in magnetic binary superlattices results in larger magnetic damping in comparison with the corresponding bulk ferromagnetic metals. In particular, a heavily enhanced damping, in comparison to bulk Co, has been observed for Co/Pt multilayers [43]. The Gilbert damping in these systems has been found, in a ferromagnetic resonance (FMR) experiment, to decrease with increasing the Co layer thickness [42]. A similar linear dependence on the inverse of the Co layer thickness has been reported for the $\mathrm{Co} / \mathrm{Pd}$ multilayers in Ref. [44]. The Gilbert damping has also been measured for $\mathrm{Co} / \mathrm{Pd}, \mathrm{Co} / \mathrm{Pt}$, and $\mathrm{Co} / \mathrm{Ni}$ multilayers by the time-resolved magneto-optical Kerr effect (TRMOKE) technique [22,23,48] and it has been found to grow linearly with increasing ratio $t_{\mathrm{NM}} / t_{\mathrm{Co}}$ for Co/NM multilayers with various Co and $\mathrm{NM}(\mathrm{Pd}$, $\mathrm{Pt}$ ) layer thicknesses, $t_{\mathrm{Co}}$ and $t_{\mathrm{NM}}$, respectively.

Despite these experimental reports, to our knowledge, there are only few theoretical papers on the Gilbert damping in FM/NM multilayers so far, and the scope of the reported calculations is rather limited. A general theoretical formalism to describe this phenomenon in magnetic multilayers, based 
on $s$ - $d$ exchange at the interfaces, has been proposed by Berger [53]. More recently, the damping constant was found numerically $[54,55]$ in bulk magnetic alloys with $\mathrm{L}_{0}$ structure within the torque-correlation model [38]. A very recent paper by $\mathrm{Qu}$ and Victora [56] reports calculations of $\alpha$ for $\mathrm{Co} / \mathrm{Pd}$ and $\mathrm{Co} / \mathrm{Pt}$ infinite superlattices with the fixed NM layer thickness of 6 monolayers (ML) and some ranges of the Co layer thickness.

In our previous works [57,58], the damping constant $\alpha$ has been calculated for bulk ferromagnets, ferromagnetic films, $\mathrm{Co} / \mathrm{NM}$ bilayers $(\mathrm{NM}=\mathrm{Cu}, \mathrm{Ag}, \mathrm{Pd}, \mathrm{Pt}$, and $\mathrm{Au})$ as well as $\mathrm{Co} / \mathrm{NM}_{1} / \mathrm{NM}_{2}$ trilayers $\left(\mathrm{NM}_{1}=\mathrm{Cu}, \mathrm{Ag}\right.$, and $\mathrm{Au}$; $\mathrm{NM}_{2}=\mathrm{Pd}$ and $\mathrm{Pt}$ ). The present manuscript is devoted to the calculation of $\alpha$ for perpendicularly magnetized Co-based superlattices with fcc structure and a finite thickness, which are made of $N$ repeated $\mathrm{Co} / \mathrm{NM}$ bilayers with the (001) surfaces/interfaces. The results are reported for two different cases: (i) $\mathrm{L}_{0}[\mathrm{Co}(1 \mathrm{ML}) / \mathrm{NM}(1 \mathrm{ML})]_{N}$ superlattices composed of alternating Co and NM atomic layers and considered to be a type of ordered alloys, and (ii) $\left[\mathrm{Co}\left(N_{\mathrm{Co}} \mathrm{ML}\right) / \mathrm{NM}\left(N_{\mathrm{NM}}\right.\right.$ $\mathrm{ML})]_{N}$ binary superlattices with arbitrary thicknesses of Co and NM layers, which include $N_{\text {Co }}$ monolayers of Co and $N_{\mathrm{NM}}$ monolayers of NM, respectively. The calculations are performed by employing the Kamberský model [38] within a realistic nine-orbital tight-binding (TB) model including the spin-orbit interaction [57].

The thicknesses of the two constituent layers and the stacking number $N$ (number of repetitions) are, alongside the energy band edge alignment of the constituent materials, important parameters in binary superlattices, or rather multilayers with superlattice structure. In the present work, we investigate the dependence of $\alpha$ on the stacking number $N$, the electron scattering rate, as well as the Co and NM film thicknesses in $[\mathrm{Co} / \mathrm{NM}]_{N}$ multilayers. It is also determined how the Gilbert damping of a FM film with a fixed thickness is affected by embedding a number of NM layers. The obtained theoretical results are compared with experimental data for similar systems. In particular, this concerns the dependence on the thickness ratio $t_{\mathrm{NM}} / t_{\mathrm{Co}}$ investigated experimentally in Refs. [22,23,48].

\section{THEORY}

The magnetization dynamics of metallic systems is described phenomenologically with the Landau-Lifshitz-Gilbert equation

$$
\frac{\partial \boldsymbol{m}}{\partial t}=-\gamma \boldsymbol{m} \times \boldsymbol{H}_{\mathrm{eff}}+\alpha \boldsymbol{m} \times \frac{\partial \boldsymbol{m}}{\partial t},
$$

where $\boldsymbol{m}$ denotes the magnetization vector (in units of the saturation magnetization $\left.M_{\mathrm{s}}\right), \gamma$ is the gyromagnetic ratio, $\boldsymbol{H}_{\text {eff }}$ is the effective magnetic field, applied externally and/or due to the intrinsic magnetic anisotropy, and $\alpha$ is the Gilbert damping constant. The two terms on the right-hand side of Eq. (1) are responsible for the precession and the relaxation of magnetization, respectively. The magnetic field $\boldsymbol{H}_{\text {eff }}$ and, consequently, the equilibrium direction of magnetization $\boldsymbol{m}$ are assumed to be along the $z$ axis perpendicular to the surface of the investigated layered systems.

Within the torque-correlation model, the following expression is found [57] for the Gilbert damping constant:

$$
\alpha=\frac{\pi}{N_{\mathrm{FM}}^{\text {tot }} \mu_{\mathrm{s}}} \sum_{n, n^{\prime}} \frac{1}{\Omega_{\mathrm{BZ}}} \int d \boldsymbol{k}\left|A_{n n^{\prime}}(\boldsymbol{k})\right|^{2} F_{n n^{\prime}}(\boldsymbol{k}),
$$

where $\mu_{\mathrm{s}}$ stands for the atomic magnetic moment (in units of the Bohr magneton $\mu_{\mathrm{B}}$ ) of the FM, $N_{\mathrm{FM}}^{\text {tot }}$ is the total number of atomic layers in the ferromagnetic parts of the system, while $\Omega_{\mathrm{BZ}}$ is the volume of the two-dimensional Brillouin zone (BZ). The integration over $\boldsymbol{k}=\left(k_{x}, k_{y}\right)$ in Eq. (2) is done over the 2D BZ with the matrix elements $A_{n n^{\prime}}(\boldsymbol{k})=\left\langle n \boldsymbol{k}\left|\left[S^{-}, H_{\mathrm{SO}}\right]\right| n^{\prime} \boldsymbol{k}\right\rangle$ of the torque due to the SO interaction $H_{\text {SO }}$ (where $S^{-}=$ $\frac{1}{2}\left(\sigma_{x}-i \sigma_{y}\right)$ is defined with the familiar Pauli matrices $\sigma_{x}$ and $\left.\sigma_{y}\right)$, and the energy factor

$$
F_{n n^{\prime}}(\boldsymbol{k})=\int_{-\infty}^{\infty} d \epsilon \eta(\epsilon) L\left(\epsilon-\epsilon_{n}(\boldsymbol{k})\right) L\left(\epsilon-\epsilon_{n^{\prime}}(\boldsymbol{k})\right) .
$$

The latter integral includes the negative derivative $\eta(\epsilon)=$ $-d f_{\mathrm{FD}} / d \epsilon$ of the Fermi-Dirac function $f_{\mathrm{FD}}(\epsilon)$ and the two Lorentz functions $L\left(\epsilon-\epsilon_{n}(\boldsymbol{k})\right), L\left(\epsilon-\epsilon_{n^{\prime}}(\boldsymbol{k})\right)$ depending on electron energies $\epsilon_{n}(\boldsymbol{k}), \epsilon_{n^{\prime}}(\boldsymbol{k})$ with band indices $n, n^{\prime}$. The Lorentzians in Eq. (3) are of the form $L(x)=(\Gamma / 2 \pi) /\left(x^{2}+\right.$ $\left.\Gamma^{2} / 4\right)$ and their full width at half maximum $\Gamma$ is the average electron scattering rate, treated here as an independent parameter. At finite temperature $T$ (entering $\eta(\epsilon)$ ), instead of a direct calculation, the integral in Eq. (3) can be efficiently replaced by a sum of Matsubara frequencies as discussed in Ref. [57]. This method is also used in the present work assuming $T=300 \mathrm{~K}$, which has previously been shown [57], for both ferromagnetic films and magnetic bilayers, to give nearly the same values of $\alpha$ as those found with $T=0$.

The expression (2) for the damping constant includes both intraband $\left(n=n^{\prime}\right)$ and interband $\left(n \neq n^{\prime}\right)$ contributions. It was recently argued by Edwards [59] that intraband contributions are absent when only the leading term of $\alpha$, quadratic in the SO coupling, is kept. However, as pointed out in Ref. [59], this argument is valid solely for systems with the inversion symmetry, like bulk cubic metals, where the diagonal elements $A_{n n}(\boldsymbol{k})$ of the spin-orbit torque vanish if electron states $|n \boldsymbol{k}\rangle$ are calculated with the zero SO coupling. The presently investigated $[\mathrm{Co} / \mathrm{NM}]_{N}$ multilayers lack such a symmetry, thus the intraband term of $\alpha$ is expected to be finite (cf. Sec. III B) and it is kept in our calculations. Let us note here that the validity of the Kamberský model is also questioned for systems without the inversion symmetry in Ref. [59], though no modification of the model has been proposed in this case and further theoretical work may be needed to clarify this point.

The formula (2) is used here to calculate $\alpha$ for the considered magnetic multilayers with several values of the scattering rate $\Gamma$ from the interval $[0.001 \mathrm{eV}, 2.0 \mathrm{eV}]$. The damping constant $\alpha$ is calculated for the region of the integration over $\boldsymbol{k}$ in Eq. (2) limited to the $1 / 8$ of the 2D BZ instead of the whole BZ which does not affect the results. The evaluation of $\alpha$ is performed within a realistic nine-orbital TB model. The TB Hamiltonian $H$, including the SO interaction term, is constructed based on the general method of Ref. [57] while the corresponding eigenstates and their energies, required for the evaluation of the expressions (2) and (3), are found numerically by the diagonalization of $H$.

The calculations are done for parallel alignment of magnetizations of all Co layers. Such an alignment is observed [6,7] in $[\mathrm{Co} / \mathrm{Pd}]_{N}$ and $[\mathrm{Co} / \mathrm{Pt}]_{N}$ multilayers, where the interlayer exchange coupling vanishes [6,7]. In the other investigated $[\mathrm{Co} / \mathrm{NM}]_{N}$ systems, with $\mathrm{NM}=\mathrm{Cu}, \mathrm{Ag}$, and $\mathrm{Au}$, this 
coupling was reported $[5,8,9,60]$ to oscillate with the NM spacer thickness and thus the antiparallel alignment occurs for some ranges of the NM layer thickness. However, the parallel alignment can still be achieved in these structures by applying a sufficiently strong magnetic field. It should also be stressed that the Kamberský model is not valid for the antiparallel alignment since the expression for $\alpha$ involves the denominator proportional to the total magnetic moment which vanishes for such a alignment $[38,58]$.

\section{RESULTS}

\section{A. $\mathrm{L1}_{\mathbf{0}}[\mathrm{Co} / \mathrm{NM}]_{N}$ superlattices}

The Gilbert damping constant $\alpha$ is calculated for $\mathrm{L}_{0}$ $[\mathrm{Co}(1 \mathrm{ML}) / \mathrm{NM}(1 \mathrm{ML})]_{N}$ superlattices $(\mathrm{NM}=\mathrm{Cu}, \mathrm{Ag}, \mathrm{Pd}$, $\mathrm{Pt}$, and $\mathrm{Au}$ ) with different electron scattering rates $\Gamma$. It is found that $\alpha$ in all the considered $\mathrm{L}_{0}$ superlattices increases with decreasing $\Gamma$ regardless of the stacking number $N$ if shallow minima which occur at $\Gamma \simeq 0.1 \mathrm{eV}$ for some film thicknesses are disregarded (this is illustrated for $N=6$ in Fig. 1). The values of $\Gamma$ corresponding to the minima of the Gilbert damping are similar as in bulk ferromagnets [61,62]. The obtained $\Gamma$ dependence of $\alpha$ as well as the prediction of larger damping in the $\mathrm{Co} / \mathrm{Pt}$ system than in the $\mathrm{Co} / \mathrm{Pd}$ one (over 3 times for both $\Gamma=0.1$ and $0.01 \mathrm{eV}$ ) are in accordance with the results previously found [55] for the Gilbert damping in $\mathrm{L}_{0} \mathrm{Co} / \mathrm{Pd}$ and $\mathrm{Co} / \mathrm{Pt}$ ordered alloys. However, the presently obtained values of $\alpha$ for these systems are smaller than the respective values reported by Victora et al. [55], which can be attributed to the significantly different TB parametrization of the electronic structure applied by those authors. It should also be noted that the systems considered in Ref. [55] are infinite $\mathrm{L}_{0}$ superlattices, corresponding to $N=\infty$.

Figure 2 presents the damping constant $\alpha$ against the stacking number $N$ in $\mathrm{L}_{0}[\mathrm{Co}(1 \mathrm{ML}) / \mathrm{NM}(1 \mathrm{ML})]_{N}$ superlattices with scattering rates $\Gamma=0.01$ and $0.1 \mathrm{eV}$. The value of $\alpha$ for $\Gamma=0.01 \mathrm{eV}$ declines in all investigated $\mathrm{L} 1_{0}$ superlattices as the thicknesses of the films $(2 N \mathrm{ML})$ increase and this trend is present even for $N=10$. The decrease of $\alpha$ in the considered $\mathrm{L} 1_{0}$ superlattices upon increasing $N$ from 1 to 10 is about tenfold for $\mathrm{Cu}$ as $\mathrm{NM}$, sixfold for $\mathrm{Pd}$, sevenfold for $\mathrm{Pt}$, and ninefold for $\mathrm{Ag}$ and $\mathrm{Au}$. The dependence of $\alpha$ on the stacking number is quite different for $\Gamma=0.1 \mathrm{eV}$ since it

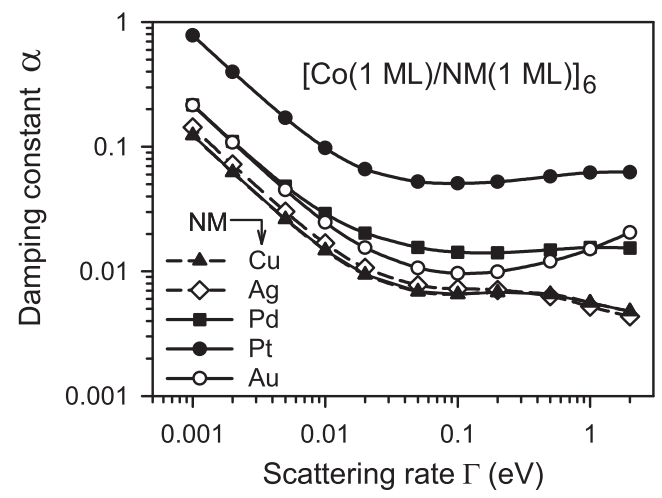

FIG. 1. Gilbert damping constant versus the scattering rate $\Gamma$ in $\mathrm{L} 1_{0}[\mathrm{Co}(1 \mathrm{ML}) / \mathrm{NM}(1 \mathrm{ML})]_{6}$ superlattices. quickly starts to saturate with increasing $N$, already at $N=3$, 4 , or 5 , depending on the type of NM.

As seen in Fig. 2, there is a noticeable difference between the dependencies of the damping constant $\alpha$ on the stacking number $N$ obtained for the binary superlattices with $\mathrm{NM}=\mathrm{Cu}$, $\mathrm{Ag}$, and $\mathrm{Au}$ and those with $\mathrm{NM}=\mathrm{Pd}$ and $\mathrm{Pt}$. In particular, a sharp fall of damping occurs on going from $N=1$ to $N=2$ in systems with $\mathrm{NM}=\mathrm{Cu}, \mathrm{Ag}$, and $\mathrm{Au}$ whereas for the $\mathrm{Co} / \mathrm{Pd}$ and $\mathrm{Co} / \mathrm{Pt}$ superlattices $\alpha$ declines more smoothly with $N$ if $\Gamma=0.01 \mathrm{eV}$ and it has the maximum value at $N=2$ for $\Gamma=0.1 \mathrm{eV}$. A possible way to account for this difference (however subtle it is) is to attribute it to the different densities of states (DOS) of the NM metals at the Fermi level $\epsilon_{\mathrm{F}}$ : low in $\mathrm{Cu}, \mathrm{Ag}, \mathrm{Au}$ and high in $\mathrm{Pd}, \mathrm{Pt}$ where the $d$ band crosses $\epsilon_{\mathrm{F}}$. This point is discussed in more detail in Sec. III B, by the analysis of the band structure at different $\mathrm{Co} / \mathrm{NM}$ interfaces in multilayers with thicker Co and NM layers.

The Gilbert damping constant is found to be largely enhanced in all investigated $[\mathrm{Co}(1 \mathrm{ML}) / \mathrm{NM}(1 \mathrm{ML})]_{N}$ superlattices in comparison with its value for bulk Co as well as with $\alpha$ found for the $\operatorname{Co}(N$ ML) film [57]. This enhancement is attributed to the large SO coupling of constituent nonmagnetic metals with $d$ states at the Fermi level (like $\mathrm{Pd}$ and $\mathrm{Pt}$ ) as well as the change of electronic structure in Co layers due to hybridization at $\mathrm{Co} / \mathrm{NM}$ interfaces. As previously found in the

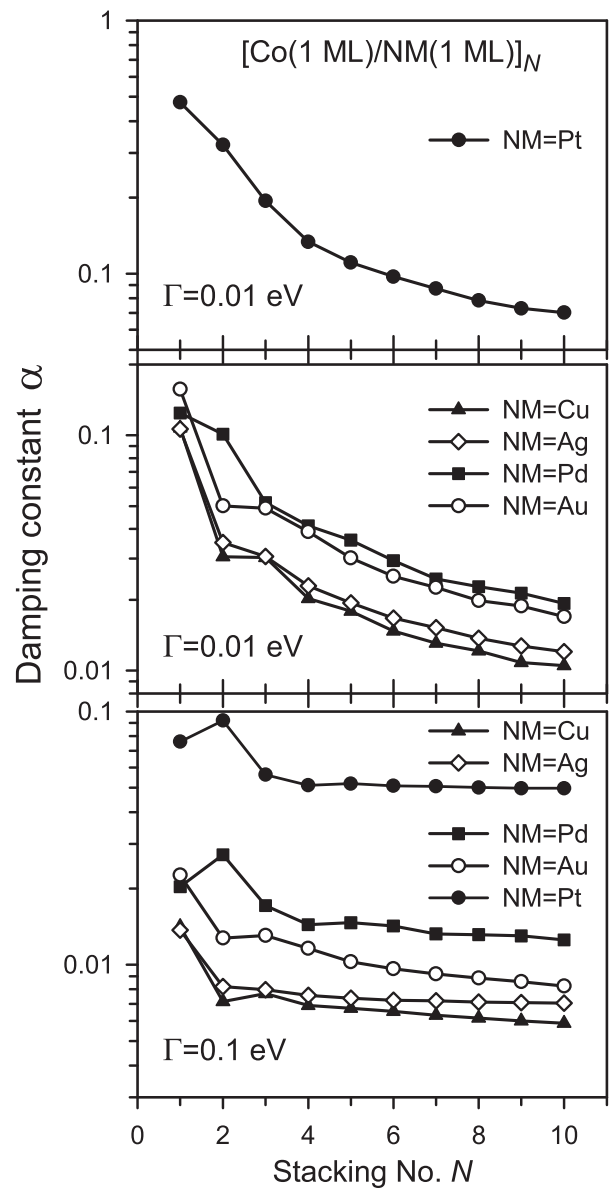

FIG. 2. Gilbert damping constant versus the stacking number $N$ in $\mathrm{L}_{0}[\mathrm{Co}(1 \mathrm{ML}) / \mathrm{NM}(1 \mathrm{ML})]_{N}$ superlattices with two different scattering rates $\Gamma=0.1$ and $0.01 \mathrm{eV}$. 
case of $\mathrm{Co} / \mathrm{NM}$ bilayers [57], also for the $\mathrm{L} 1_{0}$ superlattices one can see the particularly strong effect of the large SO coupling of $\mathrm{Pt}$, as compared to the other NM metals, in raising the Gilbert damping. This effect is also found experimentally [26] for $\mathrm{L1}_{0} \mathrm{FePd}_{1-x} \mathrm{Pt}_{x}$ superlattices (12-nm thick) where the measured damping constant strongly grows with increasing the Pt concentration $x$. The presently obtained value of $\alpha=0.051$ for $[\mathrm{Co}(1 \mathrm{ML}) / \mathrm{Pt}(1 \mathrm{ML})]_{6}$ multilayer with $\Gamma=0.1 \mathrm{eV}$ agrees very well with $\alpha=0.05$ found experimentally for a similar system $[\operatorname{Co}(0.2 \mathrm{~nm}) / \mathrm{Pt}(0.24 \mathrm{~nm})]_{6}$ sandwiched between two thick Ru layers [52]. The damping constants $\alpha$ calculated for $\mathrm{L}_{0} \mathrm{Co} / \mathrm{Pd}$ and $\mathrm{Co} / \mathrm{Pt}$ films with the scattering rate $\Gamma=0.1 \mathrm{eV}$ are 3-4 times smaller than $\alpha$ observed, correspondingly, in $\mathrm{L}_{0} \mathrm{Fe} / \mathrm{Pd}$ and FePt superlattices [26,50]. This can be related to the fact that the Gilbert damping in such Fe/Pt systems has been shown experimentally [27] to be strongly enhanced by intermixing at FM/NM interfaces which is not accounted for in the present calculations for $\mathrm{Co} / \mathrm{NM}$ superlattices. Indeed, our theoretical values of $\alpha$ in the $\mathrm{L}_{0} \mathrm{Co} / \mathrm{Pt}$ films with $\Gamma \sim 0.1 \mathrm{eV}$ are close to $\alpha \approx 0.05$ found experimentally in the $\mathrm{L} 1_{0} \mathrm{FePt}$ films with low antisite disorder [27].

The damping constant in the $[\mathrm{Co}(1 \mathrm{ML}) / \mathrm{NM}(1 \mathrm{ML})]_{N}$ superlattices (Fig. 2) is also larger than in $\operatorname{Co}(N \mathrm{ML}) / \mathrm{NM}$ bilayers, in particular in those with the same Co and NM total thicknesses (up to two times larger for systems including $6 \mathrm{ML}$ of $\mathrm{Co}$ and $6 \mathrm{ML}$ of $\mathrm{Pt}$ ). Such an enhancement can be related to the number of $\mathrm{Co} / \mathrm{NM}$ interfaces which is larger in a $[\mathrm{Co}(1 \mathrm{ML}) / \mathrm{NM}(1 \mathrm{ML})]_{N}$ superlattice than in a $\mathrm{Co} / \mathrm{NM}$ bilayer $(2 N-1$ interfaces in the superlattices compared to only one interface in the bilayers). Indeed, one can argue that in the $\mathrm{L}_{0}$ superlattices each Co atomic layer is in direct contact with a NM layer while only one Co atomic layer is adjacent to NM in the bilayers. Then, in the language of spin pumping theory, the magnetization precessing in the Co layers of a $\mathrm{L}_{0}$ superlattice pumps spin angular momentum more easily into its NM parts where it is effectively damped due to the SO coupling of NM, especially for $\mathrm{NM}=\mathrm{Pd}$ and Pt. However, the above argument based on spin pumping does not apply to multilayers including nonmagnetic metals like $\mathrm{NM}=\mathrm{Cu}, \mathrm{Ag}$, and $\mathrm{Au}$, which are poor spin sinks. In this case, the enhanced damping in the superlattices owes to the change of the electronic structure which is strongly modified by the presence of multiple $\mathrm{Co} / \mathrm{NM}$ interfaces and largely varies with the stacking number $N$, especially for small $N$. The origin of the damping enhancement emerging within the presently applied torque-correlation model is further discussed in a more general case of the $\mathrm{Co} / \mathrm{NM}$ multilayers below.

\section{B. $[\mathrm{Co} / \mathrm{NM}]_{N}$ multilayers}

The damping constant $\alpha$ is also calculated for binary superlattices with constituent metallic layers thicker than one monolayer. The calculations are done for multilayers with the fcc structure and atomically flat surfaces/interfaces parallel to the (001) plane. Experimentally, the sharp interfaces with small roughness are found in the fcc $\mathrm{Co} / \mathrm{NM}$ multilayers with the (001) surface orientation [63] in contrast to such multilayers with the (111) interfaces where strong intermixing (interfacial alloying) is observed [63-67]. Note that the biaxial strain in the (001) Co layers (leading to the tetragonally distorted fcc structure) is not accounted for in the present calculations.

\section{Dependence of Gilbert damping on scattering rate and type of nonmagnetic layers}

In Fig. 3, the damping constant $\alpha$ is plotted against the scattering rate $\Gamma$ for $[\mathrm{Co}(6 \mathrm{ML}) / \mathrm{NM}(4 \mathrm{ML})]_{4}$ multilayers with $\mathrm{NM}=\mathrm{Cu}, \mathrm{Ag}, \mathrm{Au}, \mathrm{Pd}$, and Pt. The $\Gamma$ dependence of $\alpha$ resembles the one for bulk Co [61,62] regardless of the fact that the damping is enhanced in multilayers due to the presence of the NM. In particular, the shallow minimum of the damping constant with respect to the scattering rate occurs at a value of $\Gamma$ between $0.01 \mathrm{eV}$ and $0.1 \mathrm{eV}$ though the position of this minimum significantly depends on the type of the nonmagnetic metal. It is close to $\Gamma=0.1 \mathrm{eV}$ for $\mathrm{Cu}$ and $\mathrm{Ag}, \Gamma=0.05 \mathrm{eV}$ for $\mathrm{Au}$ and $\Gamma=0.02 \mathrm{eV}$ for $\mathrm{Pd}$ and $\mathrm{Pt}$, in the considered multilayers. Furthermore, the magnitude of the Gilbert damping in the $[\mathrm{Co} / \mathrm{NM}]_{N}$ multilayers strongly depends on the type of nonmagnetic metal NM as $\alpha$ increases within the sequence $\mathrm{Cu}(\mathrm{Ag}) \rightarrow \mathrm{Au} \rightarrow \mathrm{Pd} \rightarrow \mathrm{Pt}$, while being almost identical for $\mathrm{Cu}$ and $\mathrm{Ag}$. This trend, previously found for $\mathrm{Co} / \mathrm{NM}$ bilayers [57], is clearly noticable in Fig. 3 but it is also present for the $[\mathrm{Co} / \mathrm{NM}]_{N}$ systems with the stacking number $N$ other than 4 (see Fig. 4). Such a dependence of the Gilbert damping on the type of nonmagnetic metal was also observed experimentally in magnetic layered systems [68], including $\mathrm{Co} / \mathrm{NM}$ multilayers where the damping constant was found to be larger for $\mathrm{NM}=\mathrm{Pt}$ than $\mathrm{NM}=\mathrm{Pd}$ [23]. The latter experimental finding, reproduced in our calculations, is clearly related to the fact that the SO coupling is larger in $\mathrm{Pt}$ than Pd. However, it should be noted that the obtained results for $\alpha$ do not follow the simple quadratic scaling with the SO coupling constant $\xi_{\mathrm{NM}}$ of NM which was found experimentally for $\mathrm{L}_{0} \mathrm{FePd}_{x} \mathrm{Pt}_{1-x}$ superlattices [26]. Indeed, such scaling would imply that the damping constant of a $\mathrm{Co} / \mathrm{Pt}$ multilayer to be $\xi_{\mathrm{Pt}}^{2} / \xi_{\mathrm{Pd}}^{2} \approx 8$ times larger than $\alpha$ for the corresponding $\mathrm{Co} / \mathrm{Pd}$ system while the actual scaling factor obtained in our calculations is around 3 or 4 . This results from the small differences between the valence bands in $\mathrm{Pd}$ and $\mathrm{Pt}$, although these bands are similar in the two metals. In particular, the energy splittings due to the SO coupling at the high-symmetry lines in the BZ are larger in Pt than in $\mathrm{Pd}$. However, if these splittings are neglected (by using the electronic states found with the zero SO coupling) the ratio of the damping constants calculated for $\mathrm{Co} / \mathrm{Pt}$ and $\mathrm{Co} / \mathrm{Pd}$

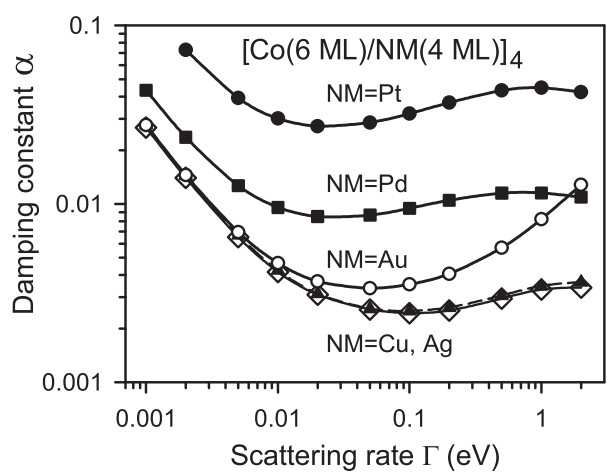

FIG. 3. Gilbert damping constant vs the scattering rate $\Gamma$ in $[\mathrm{Co}(6$ $\mathrm{ML}) / \mathrm{NM}(4 \mathrm{ML})]_{4}$ multilayers. The values of $\alpha$ for multilayers with $\mathrm{NM}=\mathrm{Cu}$ and $\mathrm{Ag}$, marked with solid triangles and open diamonds, respectively, nearly coincide. 

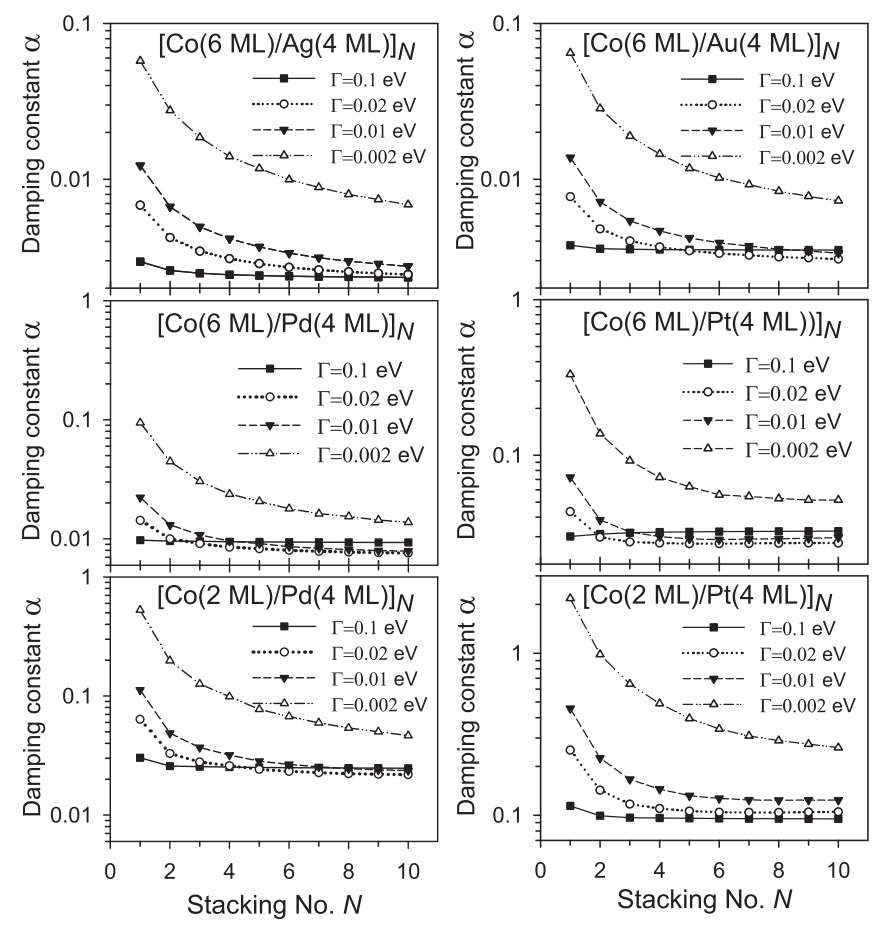

FIG. 4. Gilbert damping constant in $[\mathrm{Co} / \mathrm{NM}(4 \mathrm{ML})]_{N}$ multilayers as a function of stacking number $N$, obtained with different scattering rates $\Gamma$.

multilayers increases only to approximately 5 . Thus, other differing details of the corresponding electronic structures need to be taken into account to explain why the damping constant does not scale as $\xi_{\mathrm{NM}}^{2}$ for $\mathrm{NM}=\mathrm{Pd}$ and Pt. The quadratic scaling of $\alpha$ with $\xi_{\mathrm{NM}}$ is also not observed in the $\mathrm{Co} / \mathrm{NM}$ multilayers with the (111) interfaces since the experimental damping constant in such $\mathrm{Co} / \mathrm{Pt}$ multilayers is only twice as large as $\alpha$ measured for the Co/Pd multilayers with the same geometric structure [23]. This suggests that the magnetic damping observed in these $\mathrm{Co} / \mathrm{Pd}$ multilayers includes large contributions which do not result from the SO coupling but are due to other factors, in particular, the two-magnon scattering arising from the interface roughness and intermixing. Such explanation is supported by a recent experimental finding [27] that the damping constant $\alpha$ in $\mathrm{L} 1_{0}$ $\mathrm{Fe} / \mathrm{Pt}$ structures is strongly enhanced by intermixing leading to the antisite disorder. The relation of the Gilbert damping to the SO coupling of NM and its DOS is further discussed in view of the spatial decomposition of $\alpha$ in Sec. III C.

\section{Dependence on stacking number and comparison with experiment}

In Fig. 4, the damping constant $\alpha$ is plotted against the stacking number $N$ for $[\mathrm{Co}(2 \mathrm{ML}) / \mathrm{NM}(4 \mathrm{ML})]_{N}$ and $[\mathrm{Co}(6$ $\mathrm{ML}) / \mathrm{NM}(4 \mathrm{ML})]_{N}$ multilayers with different scattering rates $\Gamma$. The Gilbert damping appreciably declines with increasing the stacking number $N$ for a fixed $\Gamma$ smaller than $0.1 \mathrm{eV}$, especially for $\Gamma \leqslant 0.01 \mathrm{eV}$. Such a decline is found both for $\mathrm{Co} / \mathrm{NM}$ multilayers with nonmagnetic metals of $\mathrm{NM}=\mathrm{Cu}$, $\mathrm{Ag}, \mathrm{Au}$ which are poor spin sinks and those like $\mathrm{NM}=\mathrm{Pd}$ and Pt known as good spin sinks. For the scattering rates of $\Gamma=$
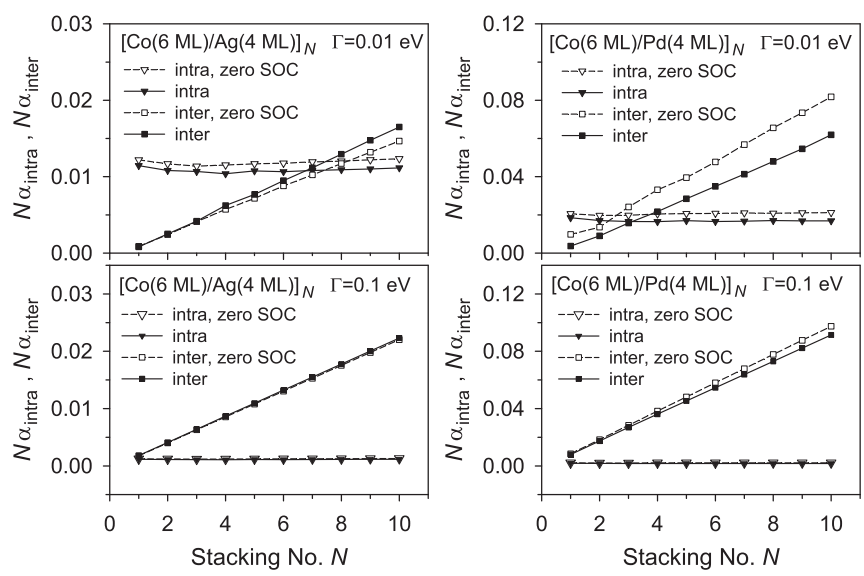

FIG. 5. Intra- and interband terms of the Gilbert damping constant in $[\mathrm{Co}(6 \mathrm{ML}) / \mathrm{Ag}(4 \mathrm{ML})]_{N}$ and $[\mathrm{Co}(6 \mathrm{ML}) / \mathrm{Pd}(4 \mathrm{ML})]_{N}$ multilayers as a function of stacking number $N$ for $\Gamma=0.01$ and $0.1 \mathrm{eV}$. The open symbols correspond to the results obtained for the electronic states calculated with the zero SO coupling.

$0.1 \mathrm{eV}$ and larger, the dependence of the damping constant on $N$ becomes very weak, and even a very slow increase of $\alpha$ with increasing $N$ is found for some of the multilayers including Pd and Pt. This implies that for large scattering rates the damping constant saturates with $N$ so quickly that it is almost equal to the damping of the basic constituent bilayer block $\mathrm{Co}\left(N_{\mathrm{Co}}\right) / \mathrm{NM}(4 \mathrm{ML})$.

The obtained approximate dependence $\alpha \approx b / N+c$, with constant $b$ and $c$, can be rewritten as $\alpha N \approx b+c N$, which clearly suggests that apart from the bulk-like damping contribution $c N$ coming from the whole volume of a multilayer there is an extra damping term $b$ originating from its parts close to the external surfaces. This conjecture is shown to be valid in all investigated $\mathrm{Co} / \mathrm{NM}$ multilayers by analyzing the distribution of atomic layer contributions to the Gilbert damping in Sec. III C. The ultimate reason for the occurrence of the surface term $b$ is the breakdown of symmetry at the surfaces, in a similar way as surface terms arise for other quantities, like magnetocrystalline anisotropy.

It is interesting to note that the constant part $c$ of $\alpha$ comes mainly from its interband term $\alpha_{\text {inter }}$ while the intraband term $\alpha_{\text {intra }}$ of the damping constant gives rise to its decaying part $b / N$, i.e., the surface term. This is well illustrated for $[\mathrm{Co} / \mathrm{Ag}]_{N}$ and $[\mathrm{Co} / \mathrm{Pd}]_{N}$ systems in Fig. 5, which shows that $N \alpha_{\text {inter }}$ grows roughly linearly with $N$ while $N \alpha_{\text {intra }}$ is almost independent of $N$. This also explains the obtained steeper decrease of $\alpha$ with $N$ for lower $\Gamma$ since $\alpha_{\text {intra }}$ grows with decreasing the scattering rate, roughly as $1 / \Gamma$. The intraband term is almost negligible for $\Gamma=0.1 \mathrm{eV}$, which explains the weak dependence of $\alpha$ on $N$ for this value of scattering rate.

As expected for systems without the inversion symmetry the intraband term is finite even if the electronic states are calculated with the zero SO coupling as proposed in Ref. [59]. In fact, the intra- and interband terms obtained with this assumption for $[\mathrm{Co} / \mathrm{Pd}]_{N}$ are larger than the corresponding terms found in the original Kamberský model using the electronic states calculated with the full SO coupling (Fig. 5). 
The predicted dependence of $\alpha$ on the stacking number $N$ is in agreement with experimental results for similar systems. For instance, the damping constant $\alpha$ is reported [51] to decline with increasing $N$ for $[\mathrm{Ni} / \mathrm{Co}]_{N}$ and $[\mathrm{Co} / \mathrm{Pd}]_{N}$ multilayers with $N \leqslant 10$, while it is found to be almost independent of the stacking number for $N \geqslant 10$. In Ref. [45], the perpendicularly magnetized $\mathrm{Co} /[\mathrm{Ni} / \mathrm{Co}]_{N}$ multilayer, sandwiched between two Pt layers, has also been investigated and the approximate dependence $\alpha \approx b / N+c$ has been observed which, therein, is attributed to the locally enhanced damping at the $\mathrm{Pt} / \mathrm{Co}$ interfaces, in agreement with our explanation given above.

However, the present results for $[\mathrm{Co}(2 \mathrm{ML}) / \operatorname{Pt}(4 \mathrm{ML})]_{N}$ do not fully agree with the experimental data reported for the multilayer $(111) \mathrm{fcc}\left[\mathrm{Co}(0.4 \mathrm{~nm}) / \mathrm{Pt}(0.8 \mathrm{~nm}]_{N}\right.$ with very similar thicknesses of $\mathrm{Co}$ and $\mathrm{Pt}$ layers [43] since the damping constant observed in experiment increases slowly with increasing $N$ (from $\alpha=0.1$ for $N=2$ to $\alpha=0.13$ for $N=12$ ). This discrepancy is not substantial since the values of $\alpha$ found for the two systems (calculated and measured, respectively) are close to each other, especially for $N \geqslant 4$. The agreement is particularly good for $\Gamma=0.1 \mathrm{eV}$ but it is also obtained for other scattering rates from the interval $0.01 \mathrm{eV} \leqslant$ $\Gamma \leqslant 0.1 \mathrm{eV}$, where $\alpha$ weakly depends on $\Gamma$. Moreover, the present results for $[\mathrm{Co}(2 \mathrm{ML}) / \mathrm{Pt}(4 \mathrm{ML})]_{N}$ and $[\mathrm{Co}(6 \mathrm{ML}) / \mathrm{Pt}(4$ $\mathrm{ML})]_{N}$ multilayers (with $N \geqslant 4$ ) obtained within the same range of $\Gamma$ agree very well with the respective values of $\alpha$ found for $x=t_{\mathrm{Pt}} / t_{\mathrm{Co}}=2.0$ and 0.67 with the approximate linear dependence on $x$ which is satisfied by the experimental data for $(111) \mathrm{fcc}\left[\mathrm{Co}\left(t_{\mathrm{Co}}\right) / \mathrm{Pt}\left(t_{\mathrm{Pt}}\right)\right]_{6}$ multilayers with different thicknesses of $\mathrm{Co}$ and Pt layers [23]. This agreement is found despite different surface orientations of the considered theoretical and experimental $\mathrm{Co} / \mathrm{Pt}$ systems. It is also worth emphasising that the mentioned range of the scattering rate includes $\Gamma \approx 0.035 \mathrm{eV}$ corresponding to the minimum of the calculated Gilbert damping in bulk Co [61] which is attained experimentally at about $T=100 \mathrm{~K}$ [69]. Larger scattering rates are expected at higher temperatures and can also account for the presence of imperfect $\mathrm{Co} / \mathrm{NM}$ interfaces in the experimental multilayers. In the recent calculations of the Gilbert damping for $\mathrm{Co} / \mathrm{NM}$ structures $\mathrm{Qu}$ and Victora $[55,56]$ assumed $\Gamma=0.1 \mathrm{eV}$ as the value which corresponds to the experimental conductivity at the room temperature. As we show in Sec. III B 4, this value of $\Gamma$ also reproduces well the experimental dependence of $\alpha$ on $x=t_{\mathrm{NM}} / t_{\mathrm{Co}}$.

In the case of the $[\mathrm{Co} / \mathrm{Pd}]_{\mathrm{N}}$ multilayers (with $N \geqslant 4$ ), the magnitude of the damping constant obtained here within the torque-correlation model for $0.01 \mathrm{eV} \leqslant \Gamma \leqslant 0.1 \mathrm{eV}$ is about 1.5-2 times lower than $\alpha$ observed experimentally for $\mathrm{Co} / \mathrm{Pd}$ multilayers [23]. To get a quantitative agreement between the experimental results reported in Ref. [23] and the calculated $\alpha$ for the $[\mathrm{Co}(2 \mathrm{ML}) / \mathrm{Pd}(4 \mathrm{ML})]_{N}$ and $[\mathrm{Co}(6 \mathrm{ML}) / \mathrm{Pd}(4 \mathrm{ML})]_{N}$ multilayers one would need to assume the respective values of $\Gamma \simeq 0.005$ and $\simeq 0.002$ in the calculations. Here, one should also note [70] that the values of the damping constant reported for $\mathrm{Co} / \mathrm{Pd}$ multilayers in Ref. [23] are more accurate than the results found by the same authors in their earlier work [22] where higher values of $\alpha$ are observed due to a lower magnitude of the external magnetic field $H_{\text {ext }}$ applied in the TRMOKE experiments which led to a higher magnetic inhomogeneity. Even lower values of $\Gamma$, much smaller than $0.002 \mathrm{eV}$ are required to get an agreement between the calculated $\alpha$ and the results of a recent experiment on $\left[\mathrm{Co}\left(\mathrm{t}_{\mathrm{Co}} \mathrm{nm}\right) / \mathrm{Pd}(0.9 \mathrm{~nm})\right]_{8}$ multilayers [44], which may be related to the fact that the TRMOKE measurements were conducted for $H_{\text {ext }}$ lower than in Ref. [23]. However, the comparison of our results with the experimental data is hindered not only by the different interface/surface orientation in the theoretical structures and the experimental multilayers but also by the fact that the $\mathrm{Co} / \mathrm{NM}$ interfaces in the experimental (111) fcc $\mathrm{Co} / \mathrm{Pd}$ and $\mathrm{Co} / \mathrm{Pt}$ layered systems exhibit significant roughness and strong intermixing [65,71]. Such structure imperfections can lead to enhancement of $\alpha$ as observed in $\mathrm{L1}_{0}$ FePt films [27] as well as an additional magnetic damping due the two-magnon scattering [40,41,72], which may become particularly significant if the external magnetic field is applied parallel or at a small angle to the film surface. The resulting extra damping terms can account for the difference between the effective Gilbert damping constant measured in experiment and the present theoretical results without resorting to the use of very small scattering rates. In particular, the net value of such additional damping terms can be estimated to be around 0.02 for $[\mathrm{Co}(2 \mathrm{ML}) / \mathrm{Pd}(4$ ML) $]_{6}$ if the Gilbert damping constant $\alpha \approx 0.03$ obtained with $\Gamma=0.1 \mathrm{eV}$ is used for comparison with experiment. If the damping contribution from the interface imperfections has a similar magnitude for $\mathrm{Co} / \mathrm{Pt}$ multilayers it is less significant than for $\mathrm{Co} / \mathrm{Pd}$ systems since it is small in comparison with the dominant term due to the spin relaxation in Pt layers with large SO coupling. It should also be noted that intermixing in $\mathrm{Co} / \mathrm{Pd}$ layered systems grows with the time elapsed from the sample fabrication [71], which is another factor that needs to be taken into account in the interpretation of the experimental results.

The Gilbert damping can also be affected by the local (intra-atomic) Coulomb interaction in NM layers, which is not included in the present TB parametrization. This interaction, responsible for the large Stoner enhancement of the magnetic susceptibility in Pd and Pt bulk metals, leads to finite magnetic moments in atomic layers of $\mathrm{NM}=\mathrm{Pd}$, $\mathrm{Pt}$ close to the $\mathrm{Co} / \mathrm{NM}$ interfaces in layered systems [73-75]. A TB model including self-consistent exchange splittings in NM atomic layers due to the Coulomb interaction has been applied in an alternative approach to the Gilbert damping based on the determination of the spin-wave spectrum directly from the transverse magnetic susceptibility [75,76]. Within this approach, it is found that the Coulomb interaction in Pd and Pt strongly modifies the damping constant in $\mathrm{Co} / \mathrm{NM}$ bilayers in the presence of the SO coupling and in particular reduces the damping by a factor of three for $\operatorname{Co}(2 \mathrm{ML}) / \mathrm{Pt}(2 \mathrm{ML})$. However, if the inclusion of this interaction had a similar effect on the Gilbert damping calculated in the Kamberský model it would make our results for $\alpha$ in $[\mathrm{Co} / \mathrm{Pd}]_{N}$ multilayers (at $\Gamma=0.1 \mathrm{eV}$ ) even smaller and thus would not diminish the discrepancy with experiment [23].

\section{Effect of embedding nonmagnetic layers}

Varying the stacking number $N$ at a fixed thickness $N_{\text {Co }}$ of Co layers leads to different total thicknesses $N_{\mathrm{Co}}^{\text {tot }}=N \times N_{\mathrm{Co}}$ of Co. On the other hand, by choosing multilayers with various values of $N_{\text {Co }}$ at fixed $N_{\text {Co }}^{\text {tot }}$ we can also investigate how embedding a number of NM layers into the Co film of the thickness $N_{\text {Co }}^{\text {tot }}$ affects its magnetic damping. This effect is illustrated in 

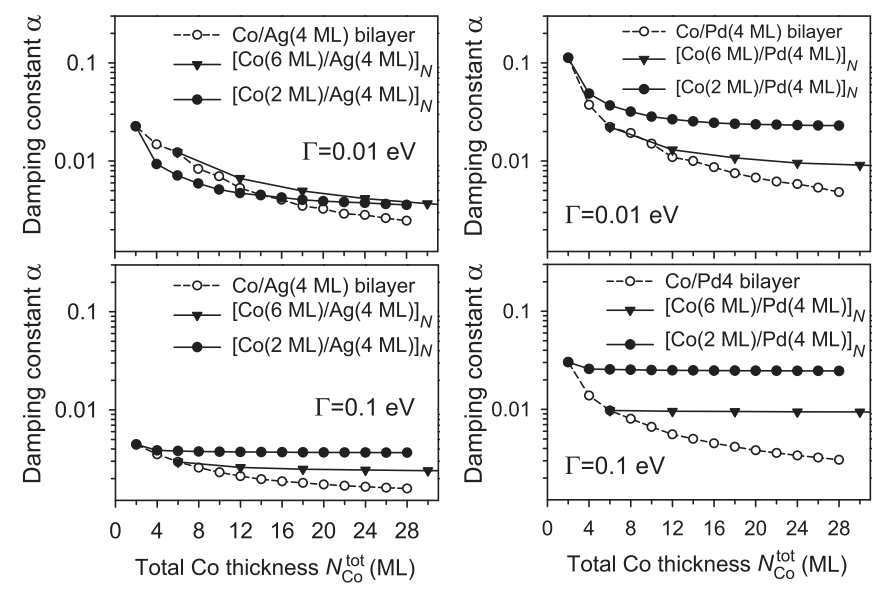

FIG. 6. Gilbert damping constant $\alpha$ vs the total Co thickness $N_{\mathrm{Co}}^{\text {tot }}=N \times N_{\mathrm{Co}}$ in $\left[\mathrm{Co}\left(N_{\mathrm{Co}}\right) / \mathrm{NM}(4 \mathrm{ML})\right]_{N}$ multilayers $(\mathrm{NM}=\mathrm{Cu}$, $\mathrm{Pd})$ with $N_{\mathrm{Co}}=2 \mathrm{ML}, 6 \mathrm{ML}$ and $\operatorname{Co}\left(N_{\mathrm{Co}}^{\mathrm{tot}}\right) / \mathrm{NM}(4 \mathrm{ML})$ bilayers for two different scattering rates $\Gamma=0.1$ and $0.01 \mathrm{eV}$.

Fig. 6 where we plot $\alpha$ in $\left[\mathrm{Co}\left(N_{\mathrm{Co}}\right) / \mathrm{NM}(4 \mathrm{ML})\right]_{N}$ multilayers with $\mathrm{NM}=\mathrm{Pd}, \mathrm{Ag}$ and $N_{\mathrm{Co}}=2,6 \mathrm{ML}$, against the total Co layer thickness for two different values of the scattering rate and compare it with the damping constant obtained for the $\operatorname{Co}\left(N_{\mathrm{Co}}^{\text {tot }}\right) / \mathrm{NM}(4 \mathrm{ML})$ bilayers. The calculated $\alpha$ decreases with increasing $N_{\mathrm{Co}}^{\text {tot }}$ in all these systems with $\Gamma=0.01 \mathrm{eV}$. For this value of $\Gamma$, the damping constant $\alpha$ is enhanced in the $\mathrm{Co} / \mathrm{NM}$ multilayers as compared to the corresponding bilayers with the same total Co thickness, though for the Co/Ag structures with $N_{\mathrm{Co}}=2 \mathrm{ML}$ this enhancement is obtained only for $N_{\mathrm{Co}}^{\text {tot }} \geqslant 16 \mathrm{ML}$ and it is smaller than for the Co/Pd systems. Thus, dividing the $\mathrm{Co}\left(N_{\mathrm{Co}}^{\text {tot }}\right)$ film into several layers of $\operatorname{Co}\left(N_{\mathrm{Co}}\right)$, separated from each other with $\mathrm{NM}(4 \mathrm{ML})$ layers, retains the general decreasing trend in the dependence of the Gilbert damping against the total Co thickness $N_{\mathrm{Co}}^{\text {tot }}$, which is also found in free-standing Co films and Co/NM bilayers with $\Gamma=0.01 \mathrm{eV}$ [57]. Simultaneously, such division enhances the damping constant in Co/NM systems and this enhancement is stronger for a finer division (i.e., larger $N$ ), leading to smaller Co layer thickness $N_{\mathrm{Co}}=N_{\mathrm{Co}}^{\text {tot }} / N$. The enhancement of $\alpha$ with $N$ for a fixed total Co thickness $N_{\mathrm{Co}}^{\text {tot }}$ is even more pronounced in the considered $\left[\mathrm{Co}\left(N_{\mathrm{Co}}\right) / \mathrm{NM}(4 \mathrm{ML})\right]_{N}$ multilayers with $\Gamma=0.1 \mathrm{eV}$. For this value of $\Gamma$ it is also found that the damping constant in these systems is almost independent of $N$, or $N_{\mathrm{Co}}^{\text {tot }}=N \times N_{\mathrm{Co}}$, at fixed $N_{\mathrm{Co}}$ (see Figs. 4 and 6).

Such an enhancement of the Gilbert damping in $\mathrm{Co} / \mathrm{Pd}$ multilayers can be attributed to the fact that for a larger number of $\mathrm{Co} / \mathrm{Pd}$ interfaces $(2 N-1)$ the spin current due to the magnetization precessing in Co layers is pumped more easily into Pd layers and dissipated there. This explanation based on the concepts of the spin pumping theory is also plausible for $\mathrm{NM}=\mathrm{Pt}$ but it cannot however apply to Co/NM multilayers with $\mathrm{NM}=\mathrm{Cu}, \mathrm{Ag}$, and $\mathrm{Au}$ which are poor spin sinks. For such systems, one can argue within the present model that it is the evolution of electronic structure in Co layers that explains the discussed enhancement of $\alpha$. The effect of introducing the $\mathrm{Co} / \mathrm{NM}$ interfaces in a Co film can be analyzed in more detail with the atomic layer contributions to the Gilbert damping which is done in Sec. III C.
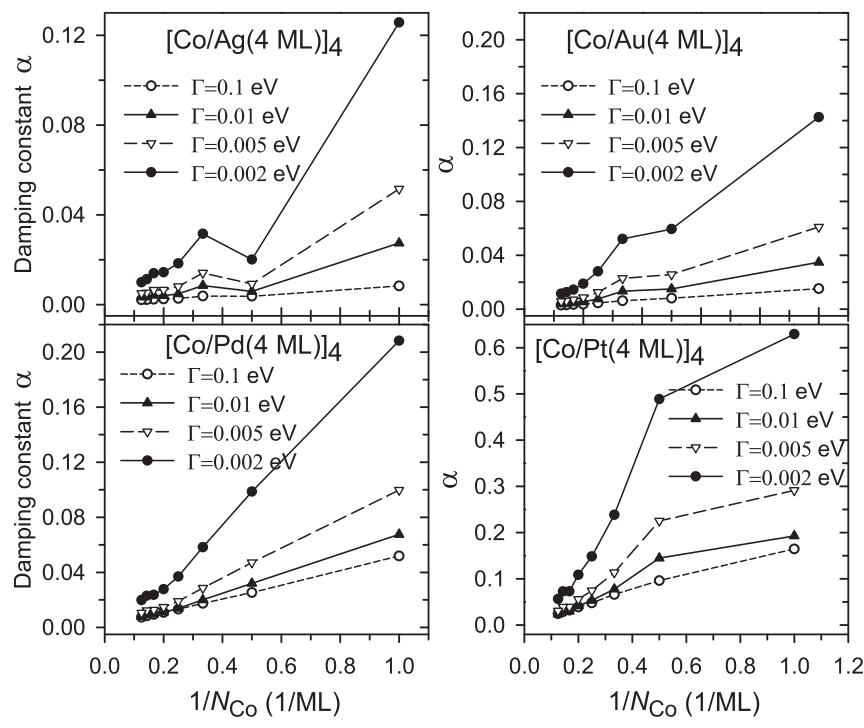

FIG. 7. Gilbert damping constant $\alpha$ vs $1 / N_{\mathrm{Co}}$ in $\left[\mathrm{Co}\left(N_{\mathrm{Co}}\right) / \mathrm{NM}(4\right.$ $\mathrm{ML})]_{4}$ multilayers with different scattering rates $\Gamma$.

\section{Dependences on Co and nonmagnetic layer thicknesses and their ratio}

In the next steps, we investigate the Gilbert damping in the $\mathrm{Co} / \mathrm{NM}$ multilayers with various Co and $\mathrm{NM}$ layer thicknesses, $N_{\mathrm{Co}}$ and $N_{\mathrm{NM}}$, respectively. As shown for $\left[\mathrm{Co}\left(N_{\mathrm{Co}} \mathrm{ML}\right) / \mathrm{Pd}(4\right.$ ML) $]_{4}$ in Fig. 7, the calculated damping constant $\alpha$ increases roughly linearly with increasing $1 / N_{\mathrm{Co}}$, while growing with decreasing the scattering rate $\Gamma$. Such an increase of $\alpha$ in the $[\mathrm{Co} / \mathrm{NM}]_{N}$ multilayer is also obtained for other NM thicknesses (not shown) as well as in the limiting case of $N=1$,i.e., for the Co/NM bilayers as reported in our previous work [57]. This increase is described more precisely with the formula $\alpha=$ $\alpha_{\text {interface }} / N_{\mathrm{Co}}+\alpha_{\mathrm{Co}}^{\text {bulk }}$, as already pointed out in Refs. [56,57], and results from the interfacial damping term $\alpha_{\text {interface }}$ due to the NM layers which dominates the Co bulk term $\alpha_{\mathrm{Co}}^{\text {bulk }}$.

The approximate linear dependence of the damping constant on $1 / N_{\mathrm{Co}}$ well agrees with the results of recent experiments on $\mathrm{Co} / \mathrm{Pd}$ and $\mathrm{Co} / \mathrm{Pt}$ multilayers $[22,23,44]$. In particular, such a dependence has been observed in experiment [44] for the $\left[\mathrm{Co}\left(\mathrm{t}_{\mathrm{Co}}\right) / \operatorname{Pd}(0.9 \mathrm{~nm})\right]_{8}$ multilayers with various Co thicknesses $t_{\text {Co }}$ from $0.22 \mathrm{~nm}$ to $1 \mathrm{~nm}$ and the Pd layer thickness $0.9 \mathrm{~nm}$, corresponding to $4.5 \mathrm{ML}$ in our $\mathrm{Co} / \mathrm{Pd}$ systems. Choosing $N=4$ in the present calculations is due to the fact that the Gilbert damping in $\left[\mathrm{Co}\left(\mathrm{N}_{\mathrm{Co}} \mathrm{ML}\right) / \mathrm{Pd}(4 \mathrm{ML})\right]_{4}$ multilayers with $\Gamma \geqslant 0.01 \mathrm{eV}$ almost saturates at $N=4$ (cf. Fig. 4) and then one can compare these results (obtained with less numerical effort than for $N=8$ ) with those investigated in the aforementioned experiments. It should also be noted that a similar dependence on the thickness $t_{\mathrm{Co}}$ of $\mathrm{Co}$ layer in $\mathrm{Co} / \mathrm{Pd}$ and $\mathrm{Co} / \mathrm{Pt}$ multilayers has also been found experimentally in the case of magnetic anisotropy energy. In these systems, the magnetic anisotropy energy (per unit volume) has the largest values at $t_{\mathrm{Co}} \approx 0.3 \mathrm{~nm}[16,44]$, while for thicker Co layers it declines with increasing $t_{\mathrm{Co}}$ as a linear function of $1 / t_{\mathrm{Co}}[22,23]$, which can be attributed to the interfacial magnetocrystalline anisotropy terms. 

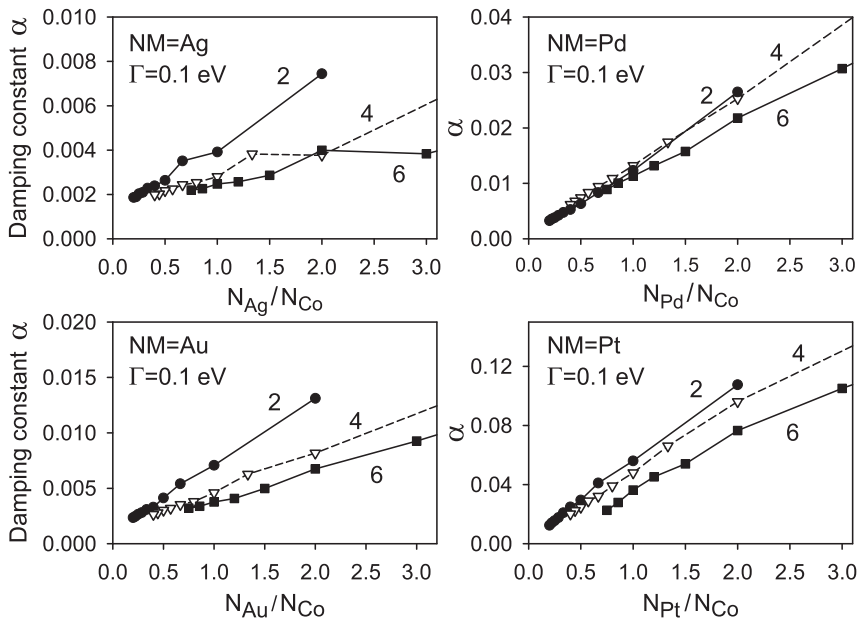

FIG. 8. Gilbert damping constant in $[\mathrm{Co} / \mathrm{NM}]_{4}$ multilayers versus the ratio $N_{\mathrm{NM}} / N_{\mathrm{Co}}$ for several thicknesses of nonmagnetic layer ( $N_{\mathrm{NM}}=2,4,6$, marked next to the respective line) and the scattering rate $\Gamma=0.1 \mathrm{eV}$.

In the experiments on $\mathrm{Co} / \mathrm{Pd}$ and $\mathrm{Co} / \mathrm{Pt}$ multilayers reported in Refs. [22,23], the damping constant $\alpha$, measured in $\left[\mathrm{Co}\left(\mathrm{t}_{\mathrm{Co}} \mathrm{nm}\right) / \mathrm{Pd}\left(\mathrm{t}_{\mathrm{Pd}} \mathrm{nm}\right)\right]_{6}$ multilayers with various Co thicknesses $t_{\mathrm{Co}}$ and three different NM thicknesses $t_{\mathrm{NM}}(0.8,1.0$, $1.5 \mathrm{~nm}$ for Pd and $0.5,1.0,1.5 \mathrm{~nm}$ for $\mathrm{Pt}$ ), was found to follow the same linear dependence on the ratio $x=t_{\mathrm{NM}} / t_{\mathrm{Co}}$. The linear dependence of $\alpha$ on $x=N_{\mathrm{NM}} / N_{\mathrm{Co}}$ is also found to be similar for different NM thicknesses $(2,4,6 \mathrm{ML})$ in the present calculations for $[\mathrm{Co} / \mathrm{Pd}]_{N}$ and $[\mathrm{Co} / \mathrm{Pt}]_{N}$ multilayers with fixed $N$ for $\Gamma=0.1 \mathrm{eV}$; see Fig. 8. The slope in the obtained $\alpha$ versus $N_{\mathrm{NM}} / N_{\mathrm{Co}}$ dependence is larger for $\mathrm{Co} / \mathrm{Pt}$ multilayers than for $\mathrm{Co} / \mathrm{Pd}$ multilayers, in agreement with experiment. This property results from the stronger SO coupling in $\mathrm{Pt}$ than $\mathrm{Pd}$, which generally agrees with the explanation given in Ref. [23] where it is attributed to the difference between the spin-diffusion lengths of the two metals.
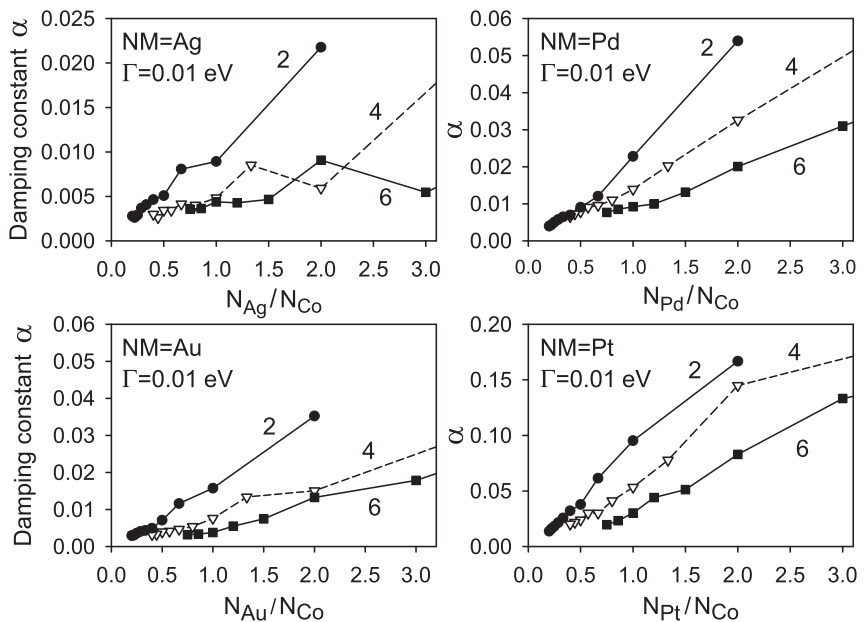

FIG. 9. Gilbert damping constant in $[\mathrm{Co} / \mathrm{NM}]_{4}$ multilayers vs the ratio $N_{\mathrm{NM}} / N_{\mathrm{Co}}$ for several thicknesses of nonmagnetic layer $\left(N_{\mathrm{NM}}=\right.$ 2,4 , and 6 , marked next to the respective line) and the scattering rate $\Gamma=0.01 \mathrm{eV}$.

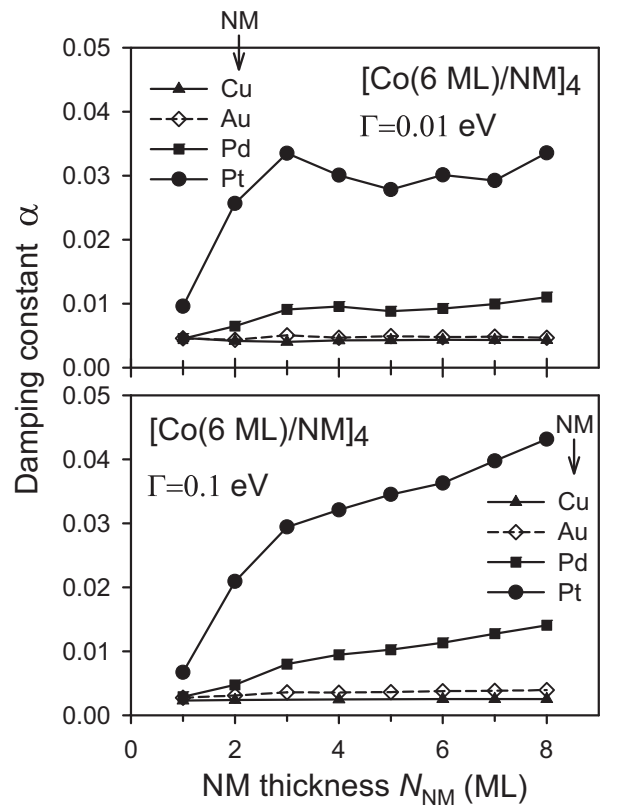

FIG. 10. Gilbert damping constant in $[\mathrm{Co}(6 \mathrm{ML}) / \mathrm{NM}]_{4}$ multilayers vs the thickness of NM, obtained for two different scattering rates $\Gamma=0.1$ and $0.01 \mathrm{eV}$.

However, the obtained slope of $\alpha$ versus $N_{\mathrm{NM}} / N_{\mathrm{Co}}$ considerably changes with the NM thickness for the $[\mathrm{Co} / \mathrm{NM}]_{N}$ multilayers with $\mathrm{NM}=\mathrm{Cu}, \mathrm{Ag}$ and $\mathrm{Au}$ for the two considered values of $\Gamma=0.01$ and $0.1 \mathrm{eV}$ as well as for the $[\mathrm{Co} / \mathrm{Pd}]_{N}$ and $[\mathrm{Co} / \mathrm{Pt}]_{N}$ multilayers with $\Gamma=0.01 \mathrm{eV}$. In fact, it is found for the $\mathrm{Co} / \mathrm{NM}$ multilayers with this lower scattering rate that $\alpha$ increases with decreasing the NM thickness at a constant value of the ratio $N_{\mathrm{NM}} / N_{\mathrm{Co}}$ (see Fig. 9). Thus, in order to reach a satisfactory agreement between the theoretical and experimental trends in the dependence of the Gilbert damping on this thickness ratio, a larger value of $\Gamma$ close to $0.1 \mathrm{eV}$ should be assumed.

The dependence of the damping constant $\alpha$ on the thickness of $\mathrm{NM}$ is further investigated for $[\mathrm{Co}(6 \mathrm{ML}) / \mathrm{NM}]_{4}$ multilayers with two different scattering rates $\Gamma=0.01$ and $0.1 \mathrm{eV}$ and it is shown in Fig. 10. It is found that $\alpha$ remains almost unaffected by changing the NM thickness for the nonmagnetic metals of $\mathrm{NM}=\mathrm{Cu}, \mathrm{Ag}$, and $\mathrm{Au}$ in which the $d$-band is below the Fermi level. For the two other metals $\mathrm{NM}=\mathrm{Pd}, \mathrm{Pt}$, the damping constant obtained for $\Gamma=0.01 \mathrm{eV}$ is found to initially grow with increasing the NM thickness and saturate as the NM thickness further increases while $\alpha$ obtained for $\Gamma=0.1 \mathrm{eV}$ grows and does not saturate within the whole investigated range of the NM thickness (up to $8 \mathrm{ML}$ ). A similar NM-thickness dependence of $\alpha$ calculated with $\Gamma=0.01 \mathrm{eV}$ was previously obtained for $\mathrm{Co} / \mathrm{NM}$ bilayers [57]. Such dependence was recently found also experimentally for $\mathrm{Co} / \mathrm{Pt}$ and $\mathrm{Co} / \mathrm{Au}$ bilayers [67].

\section{Layer contributions to Gilbert damping as tool to investigate its dependence on multilayer structure}

A deeper understanding of the Gilbert damping in $[\mathrm{Co} / \mathrm{NM}]_{N}$ multilayers, including its enhancement due to the presence of NM and the obtained dependences on their 

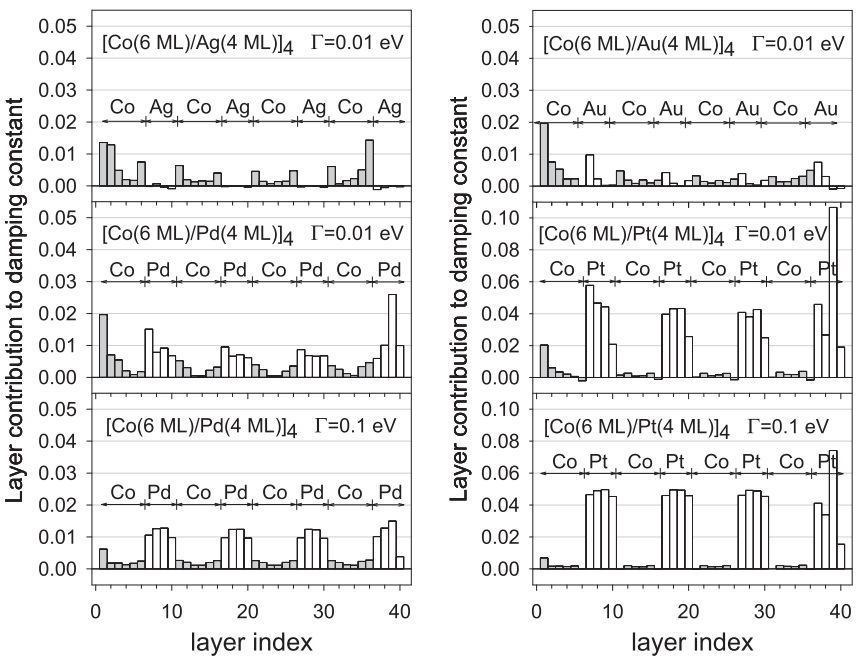

FIG. 11. Layer contributions to the damping constant $\alpha$ in $[\mathrm{Co}(6$ $\mathrm{ML}) / \mathrm{NM}(4 \mathrm{ML})]_{4}$ multilayers $(\mathrm{NM}=\mathrm{Ag}, \mathrm{Au}, \mathrm{Pd}$, and $\mathrm{Pt}) ; \Gamma=0.1$ and $0.01 \mathrm{eV}$.

geometrical parameters, can be achieved by analyzing its spatial distribution. For this purpose, the damping constant is represented as the sum of contributions $\alpha_{l}$ from individual atomic layers $l$,

$$
\alpha=\frac{1}{N_{\mathrm{FM}}^{\text {tot }}} \sum_{l=1}^{N_{\text {tot }}} \alpha_{l},
$$

where $N_{\text {tot }}$ is the total number of atomic layers. The explicit formula for the layer damping contributions $\alpha_{l}$ is given in Ref. [57], in terms of electronic states and their energies via factors similar to those appearing in Eqs. (2) and (3). The contributions $\alpha_{l}$ are not normalized and need to be divided by the total number of ferromagnetic Co layers, $N_{\mathrm{FM}}^{\text {tot }}=N_{\mathrm{Co}}^{\mathrm{tot}}=$ $N \times N_{\text {Co }}$, to give the total damping constant $\alpha$ when they are summed up. This convention is chosen since it allows for direct comparison of $\alpha_{l}$ in layered systems with different $N_{\mathrm{FM}}^{\mathrm{tot}}$. Such a method of the spatial decomposition of the Gilbert damping does not guarantee that the layer contributions $\alpha_{l}$ are positive throughout the film despite the fact that the total $\alpha$ is positive. However, as seen in the following plots (Figs. 11, 13-16), the calculated $\alpha_{l}$ are positive in almost all atomic layers while the few remaining $\alpha_{l}$ with the negative sign are very small and thus practically negligible.

The applied method of the spatial decomposition is different from the approach proposed by $\mathrm{Qu}$ and Victora in Ref. [56] where each layer contribution $\alpha_{l}^{\mathrm{QV}}$ is found with Eq. (2) modified by setting the SO coupling strength $\xi_{l^{\prime}}$ to zero in all layers $l^{\prime}$ except for one layer $l^{\prime}=l$. However, the latter method has a major drawback: the contributions $\alpha_{l}^{\mathrm{QV}}$ do not sum up to the total $\alpha$. Indeed, $\alpha_{l}^{\mathrm{QV}}$ is proportional to $\xi_{l}^{2}$ so the sum of $\alpha_{l}^{\mathrm{QV}}$ does not include terms proportional to $\xi_{l} \xi_{l^{\prime}}$, which are present in the expression (2) for $\alpha$ (cf. Ref. [57]). The layer contributions $\alpha_{l}$ adopted in this work satisfy the required sum rule provided that the scaling factor present in Eq. (4) is accounted for.

The breakdown of the Gilbert damping into layer contributions $\alpha_{l}$ was previously applied [57] to ferromagnetic films and
$\mathrm{Co} / \mathrm{NM}$ bilayers for which it has been demonstrated that the magnetic damping is most pronounced near the interfaces in $\mathrm{Co} / \mathrm{NM}$ bilayers. In particular, it has been found for the bilayers with $\mathrm{NM}=\mathrm{Pd}, \mathrm{Pt}$ and the low scattering rate of $\Gamma=0.01 \mathrm{eV}$ that the majority of damping originates from a few atomic layers of the NM cap that are closest to the Co/NM interface. In this section, the layer contributions $\alpha_{l}$ to the Gilbert damping are calculated for $\left[\mathrm{Co}\left(N_{\mathrm{Co}} \mathrm{ML}\right) / \mathrm{NM}\left(N_{\mathrm{NM}} \mathrm{ML}\right)\right]_{N}$ multilayers with different stacking numbers $N$ and various thicknesses of Co and NM layers.

The damping contributions in $[\mathrm{Co}(6 \mathrm{ML}) / \mathrm{NM}(4 \mathrm{ML})]_{4}$ multilayers $(\mathrm{NM}=\mathrm{Ag}, \mathrm{Au}, \mathrm{Pd}$, and $\mathrm{Pt})$ are shown in Fig. 11. As seen, in multilayers with $\mathrm{NM}=\mathrm{Ag}$, the Gilbert damping comes almost entirely from the ferromagnetic layers of Co and there are almost no contributions from the Ag parts. Very similar damping distributions (not shown) are found for $\mathrm{NM}=\mathrm{Cu}$ instead of $\mathrm{Ag}$. In the case of systems with $\mathrm{NM}=\mathrm{Au}$, alongside the contributions from Co layers, there are significant contributions from the interface $\mathrm{Au}$ atomic layers while the predominant layer contributions $\alpha_{l}$ in multilayers including the nonmagnetic metals of $\mathrm{NM}=\mathrm{Pd}$ and $\mathrm{Pt}$ come from the NM parts. They are particularly large, in comparison with contributions from Co parts, in multilayers with $\mathrm{NM}=\mathrm{Pt}$, much larger than in those with $\mathrm{NM}=\mathrm{Au}$, despite the very similar strengths of the SO coupling in the two nonmagnetic metals. A similar spatial distribution was reported for the layer damping contributions $\alpha_{l}^{\mathrm{QV}}$ in the $\mathrm{Co} / \mathrm{Pd}$ and $\mathrm{Co} / \mathrm{Pt}$ infinite superlattices [56], however, they significantly differ in value from $\alpha_{l}$ and have a stronger variation with $l$ in the NM layers. These differences inevitably result not only from the differing definitions of $\alpha_{l}$ and $\alpha_{l}^{\mathrm{QV}}$ but also from the different parametrizations of the TB Hamiltonian.

The SO coupling is not the sole factor affecting the Gilbert damping. Another important factor that determines the magnetic damping and its spatial distribution is the number of quantum states which have energies close to the Fermi level $\epsilon_{\mathrm{F}}$ (i.e., differing from $\epsilon_{\mathrm{F}}$ by less than a few $\Gamma$ ) and thus contribute significantly to $\alpha$ expressed with Eqs. (2) and (3).

The calculated layer-projected DOS in Co and NM atomic layers at one of the successive $\mathrm{Co} / \mathrm{NM}$ interfaces in $[\mathrm{Co}(4$ $\mathrm{ML}) / \mathrm{NM}(6 \mathrm{ML})]_{4}$ multilayers (in layers $l=16$ and 17 , where $l=1, \ldots, 40$ counts consecutive atomic layers) is shown in Fig. 12. In the ferromagnetic (Co) part there are $d$ states close to the Fermi energy $\epsilon_{\mathrm{F}}$ which leads to high DOS at $\epsilon_{\mathrm{F}}$ and results in sizable contributions to the damping from Co. The $d$ states in the vicinity of $\epsilon_{\mathrm{F}}$, manifesting themselves as large layer-projected DOS, are also present in the nonmagnetic layers of $\mathrm{NM}=\mathrm{Pd}$ and $\mathrm{Pt}$ and, consequently, they give significant, or even dominant (for $\mathrm{NM}=\mathrm{Pt}$ ), contributions to the Gilbert damping from these layers in the $\mathrm{Co} / \mathrm{Pd}$ and $\mathrm{Co} / \mathrm{Pt}$ systems. In $[\mathrm{Co} / \mathrm{NM}]_{N}$ multilayers with $\mathrm{NM}=\mathrm{Cu}$, $\mathrm{Ag}$, however, the peaks of the DOS occur at energies well below $\epsilon_{\mathrm{F}}$ since the top of the $d$ band is below $\epsilon_{\mathrm{F}}$. Thus, for the nonmagnetic metals of $\mathrm{NM}=\mathrm{Cu}, \mathrm{Ag}$, with few states in the vicinity of $\epsilon_{\mathrm{F}}$ and the SO coupling of moderate strength, the contributions to the damping from the nonmagnetic part on the $[\mathrm{Co} / \mathrm{NM}]_{N}$ multilayers are marginal. In such systems, the modification of $\alpha$ results from the change of electronic structure in Co atomic layers due to the adjacent NM layers, 

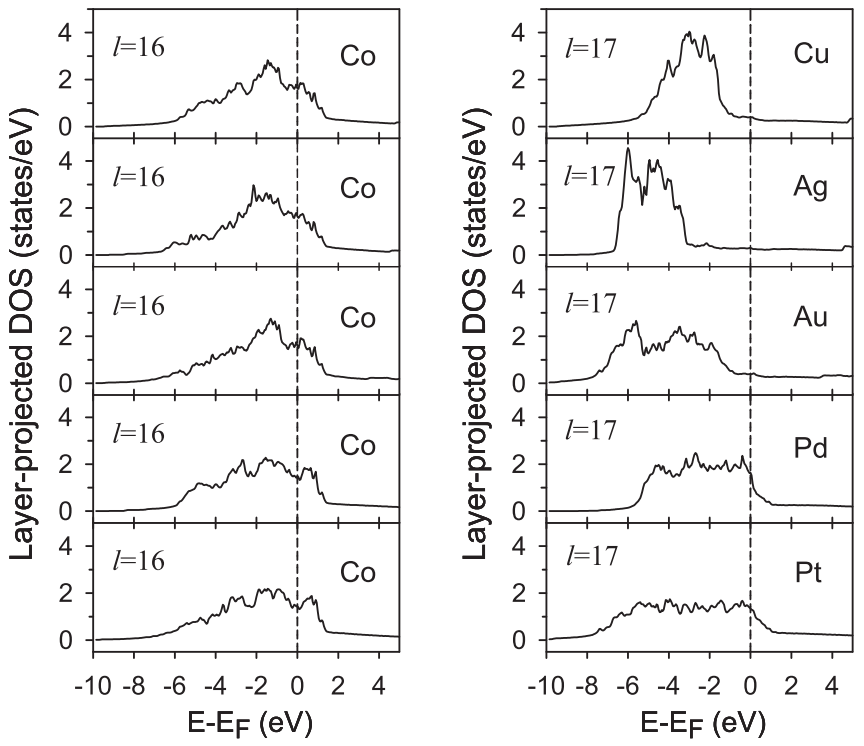

FIG. 12. Layer-projected density of states in two adjacent atomic layers $l=16$ (Co layer) and $l=17$ (NM layer) at one of the Co/NM interface of $[\mathrm{Co}(6 \mathrm{ML}) / \mathrm{NM}(4 \mathrm{ML})]_{4}$ multilayer. The vertical dashed lines mark the Fermi energy $\epsilon_{\mathrm{F}}$.

like in $\mathrm{Co} / \mathrm{NM}$ bilayers [57]. The borderline case of $\mathrm{NM}=\mathrm{Au}$ with the $d$-band below $\epsilon_{\mathrm{F}}$ but with strong SO coupling leads to significant contributions from both $\mathrm{Co}$ and $\mathrm{Au}$ atomic layers near the $\mathrm{Co} / \mathrm{Au}$ interface, as it is seen in Fig. 11. Thus the way the nonmagnetic layers influence the Gilbert damping is the combined effect of their DOS at $\epsilon_{\mathrm{F}}$ and their SO coupling strength. This, in particular, explains the obtained sequence $\mathrm{Cu}(\mathrm{Ag}) \rightarrow \mathrm{Au} \rightarrow \mathrm{Pd} \rightarrow \mathrm{Pt}$ in the increasing trend of the damping constant against the type of $\mathrm{NM}$ in $\mathrm{Co} / \mathrm{NM}$ multilayers.

The breakdown of the damping constant into its layer contributions can further be employed to investigate the mechanism of its enhancement due to embedding nonmagnetic layers of $\mathrm{NM}$ in a Co film as shown in Fig. 6. Large contributions from Pd and Pt layers as well as modified contributions from Co layers, despite very small contributions from $\mathrm{Cu}$ and $\mathrm{Ag}$ layers, can well explain this mechanism. This is illustrated in Fig. 13, which shows the layer contributions $\alpha_{l}$ calculated for $\left[\mathrm{Co}\left(N_{\mathrm{Co}}\right) / \mathrm{NM}(4 \mathrm{ML})\right]_{N}(\mathrm{NM}=\mathrm{Ag}, \mathrm{Pd}$ and $N=2,3,4$, and 6) with the fixed total number $N_{\mathrm{Co}}^{\text {tot }}=12 \mathrm{ML}$ of Co atomic layers. As seen, embedding the Pd layers inside a $\operatorname{Co}\left(N_{\mathrm{Co}}^{\text {tot }}\right.$ ML) film results in new regions where the magnetization is effectively damped thus the damping is enhanced if the number of these layers increases. The same scenario applies to embedding layers of other NM, like Pt (not shown), which are good spin sinks.

In the case of $[\mathrm{Co} / \mathrm{Ag}]_{N}$ multilayers with a fixed $N_{\mathrm{Co}}^{\text {tot }}$, one can argue that the enhancement of $\alpha$ with increasing the number of embedded $\mathrm{Ag}(4 \mathrm{ML})$ layers arises due to the contributions from $\mathrm{Co}$ atomic layers at the $\mathrm{Co} / \mathrm{Ag}$ interfaces. These contributions are much larger than the contributions from atomic layers in the $\mathrm{Ag}$ parts and the interior of Co parts, while their number increases with increasing the number of $\mathrm{Co} / \mathrm{Ag}$ interfaces. Such mechanism is clearly visible in Fig. 13 for $\Gamma=0.1 \mathrm{eV}$, while its action is disturbed in the spatial
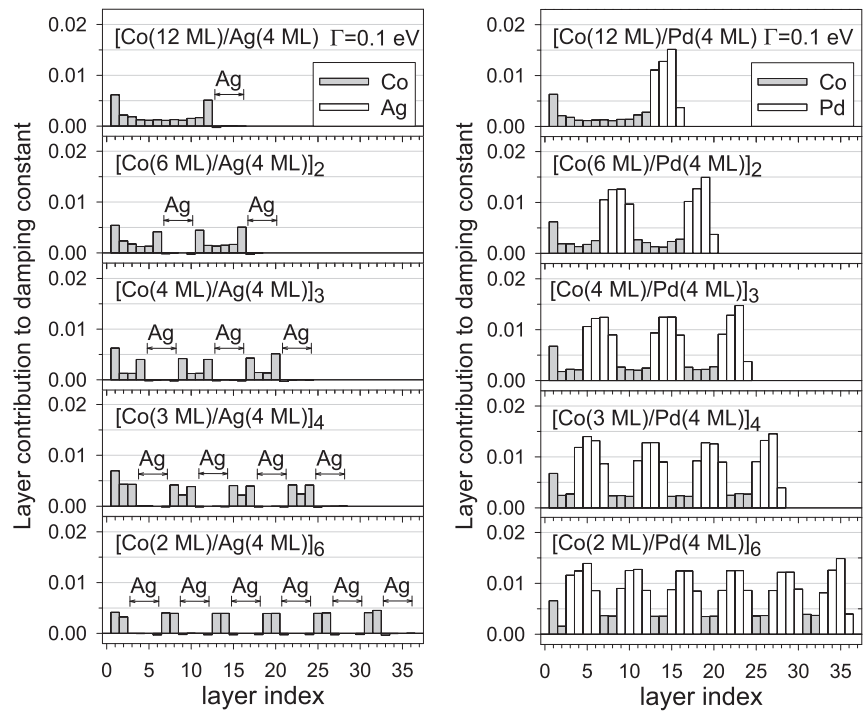

FIG. 13. Layer contributions to the damping constant $\alpha$ in $\left[\mathrm{Co}\left(N_{\mathrm{Co}} \mathrm{ML}\right) / \mathrm{NM}(4 \mathrm{ML})\right]_{N}$ multilayers $(\mathrm{NM}=\mathrm{Ag}, \mathrm{Pd})$ with the total Co thickness $N_{\mathrm{Co}}^{\text {tot }}=12 \mathrm{ML}$ and $\Gamma=0.1 \mathrm{eV}$.

damping distribution for $\Gamma=0.01 \mathrm{eV}$ (not shown) which explains why for this value of scattering rate the discussed enhancement of $\alpha$ in $[\mathrm{Co} / \mathrm{Ag}]_{N}$ multilayers occurs for $N_{\mathrm{Co}}^{\text {tot }}$ larger than $16 \mathrm{ML}$ (cf. Fig. 6).

In the next step, we use the spatial distribution of the Gilbert damping to better understand the decrease of $\alpha$ with increasing the stacking number $N$ for the $[\mathrm{Co} / \mathrm{NM}]_{N}$ multilayers with fixed thicknesses of Co and NM layers (see Fig. 4). As already noted in Sec. III B, this trend is well described with the approximate formula $\alpha \approx b / N+c$. The $b / N$ term of the damping constant arises since the damping contributions from Co and NM atomic layers close to the two outer surfaces of the multilayer are different from the contributions from the corresponding atomic layers in the Co/NM bilayer blocks located in the interior of the multilayer (see Fig. 11). Indeed, in such a case, the damping constant can be represented as

$$
\alpha=\frac{1}{N_{\mathrm{Co}}^{\mathrm{tot}}}\left(\sum_{l=1}^{N_{\mathrm{tot}}} \alpha_{l}^{\infty}+\sum_{l=1}^{N_{\mathrm{tot}}} \Delta \alpha_{l}\right),
$$

where the contributions $\alpha_{l}^{\infty}$ correspond to the infinite $\mathrm{Co} / \mathrm{NM}$ superlattice $(N=\infty)$ and they differ significantly from the corresponding layer contributions $\alpha_{l}$ in a finite $[\mathrm{Co} / \mathrm{NM}]_{N}$ multilayer only in two localized regions close to its outer surfaces; this difference is denoted by $\Delta \alpha_{l}=\alpha_{l}-\alpha_{l}^{\infty}$. Thus the sum $\Delta \alpha_{\text {surf }}=\sum_{l=1}^{N_{\text {tot }}} \Delta \alpha_{l}$ quickly saturates with increasing $N$ and, if divided by $N_{\mathrm{Co}}^{\text {tot }}=N \times N_{\mathrm{Co}}$, yields the $b / N$ term of $\alpha$ where $b=\Delta \alpha_{\text {surf }} / N_{\mathrm{Co}}$. As a result, we finally obtain the expected relation

$$
\alpha=\frac{1}{N_{\mathrm{Co}}}\left(\alpha_{\mathrm{Co}}^{\infty}+\alpha_{\mathrm{NM}}^{\infty}\right)+\frac{1}{N_{\mathrm{Co}}^{\text {tot }}} \Delta \alpha_{\text {surf }}=c+\frac{b}{N},
$$

where $\alpha_{\mathrm{Co}}^{\infty}$ and $\alpha_{\mathrm{NM}}^{\infty}$ are the damping contributions $\alpha_{l}^{\infty}$ summed over the $N_{\mathrm{Co}}$ and $N_{\mathrm{NM}}$ atomic layers of Co and NM, respectively, inside a single $\mathrm{Co} / \mathrm{NM}$ bilayer block of an infinite superlattice. 

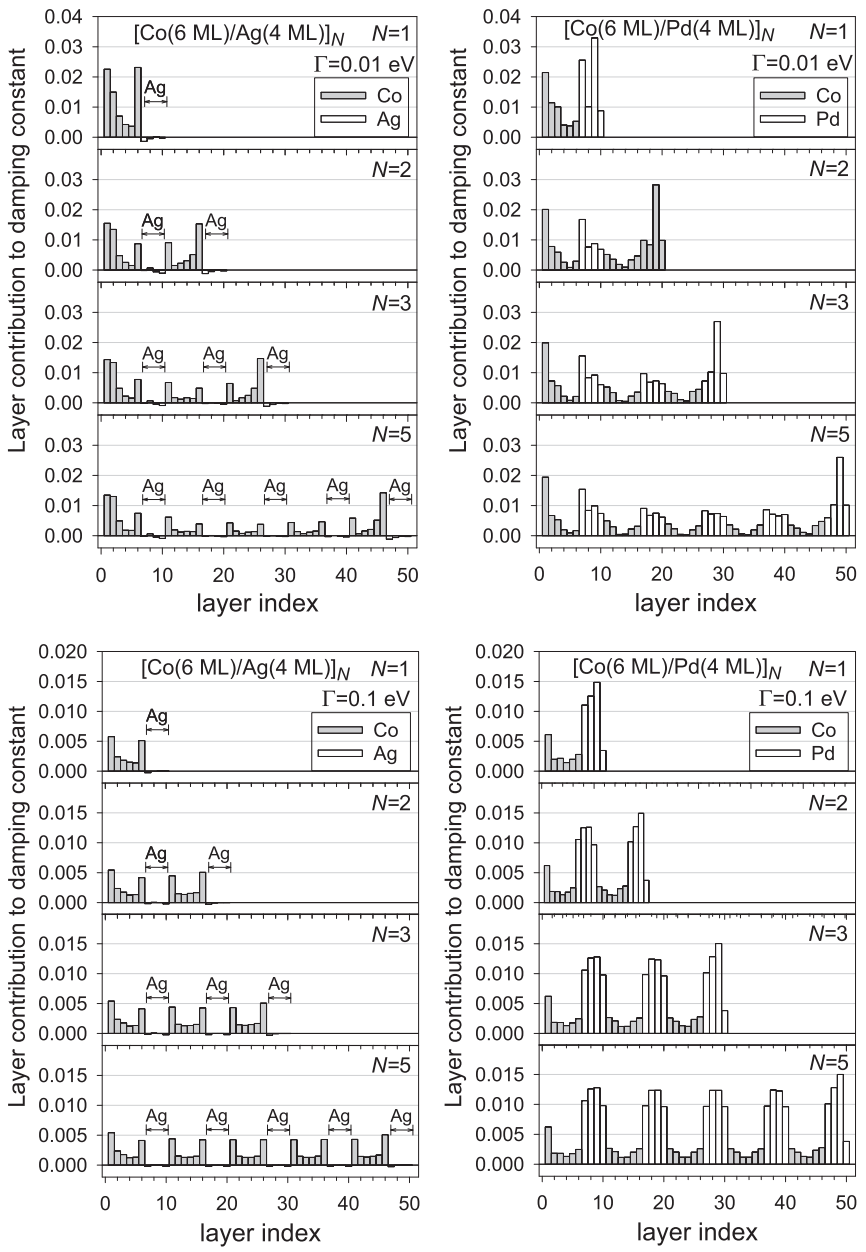

FIG. 14. Layer contributions to the damping constant $\alpha$ in $[\mathrm{Co}(6$ $\mathrm{ML}) / \mathrm{NM}(4 \mathrm{ML})]_{N}$ multilayers $(\mathrm{NM}=\mathrm{Ag}, \mathrm{Pd}) ; \Gamma=0.1$ and $0.01 \mathrm{eV}$.

Figure 14 provides a vivid picture which clearly supports the above explanation of the approximate dependence of $\alpha$ on the stacking number $N$. As seen, the distribution of the Gilbert damping is very similar in the consecutive $\mathrm{Co} / \mathrm{NM}$ bilayer blocks in the interior of the $[\mathrm{Co}(6 \mathrm{ML}) / \mathrm{NM}(4 \mathrm{ML})]_{N}$ multilayer but it is substantially altered in a few atomic layers near each of the two outer surfaces. This gives rise to the $c$ and $b / N$ terms of the damping constant but to obtain this result one also needs to note that the patterns of damping contributions in the internal $\mathrm{Co} / \mathrm{NM}$ blocks and the subsurface regions barely change with increasing $N$. The weaker $N$ dependence of $\alpha$ for larger $\Gamma$ (see Fig. 4) is in agreement with this picture since the damping contributions for $\Gamma=0.1 \mathrm{eV}$ (the lower panel of Fig. 14) are modified in a smaller number of atomic layers near the outer surfaces than the contributions for $\Gamma=0.01 \mathrm{eV}$ (the upper panel of Fig. 14), which makes $b=\Delta \alpha_{\text {surf }} / N_{\text {Co }}$ smaller for $\Gamma=0.1 \mathrm{eV}$.

The $b / N$ term determines the monotonic trend in the $N$ dependence of the damping constant $\alpha$. This term can be strongly affected by the substrate and the cap present in the experimental samples, which may even change the sign of this term. Indeed, the damping contributions from the atomic layers close to the multilayer surfaces, exposed to vacuum in our calculations, are expected to change significantly when these surfaces get in contact with the substrate and the cap
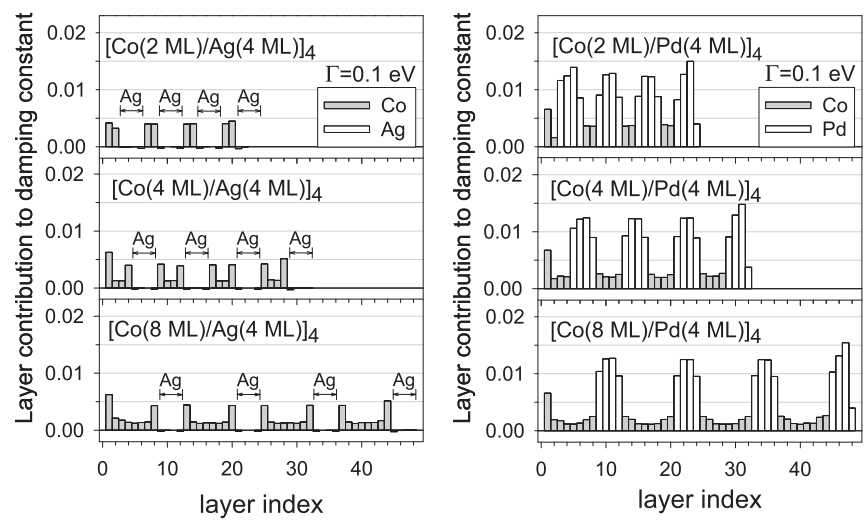

FIG. 15. Layer contributions to the damping constant $\alpha$ in $\left[\mathrm{Co}\left(N_{\mathrm{Co}} \mathrm{ML}\right) / \mathrm{NM}(4 \mathrm{ML})\right]_{4}$ multilayers $(\mathrm{NM}=\mathrm{Ag}, \mathrm{Pd})$ with $N_{\mathrm{Co}}=$ 2,4 , and $8 \mathrm{ML}$ with $\Gamma=0.1 \mathrm{eV}$. The layer contributions for $N_{\text {Co }}=6 \mathrm{ML}$ are shown in Fig. 11 .

in an experimental setup. In addition, the substrate and the cap themselves can also contribute substantially to the $b / N$ term of the damping constant if they are good spin sinks. This can lead to discrepancy between the trends in theoretical and experimental $\alpha$-versus- $N$ dependencies, like that described in Sec. III B 2.

The spatial distribution of the Gilbert damping also explains the approximate $1 / N_{\mathrm{Co}}$ dependence of the damping constant on the Co layer thickness $N_{\text {Co }}$. Such a dependence emerges since the dominating damping contributions $\alpha_{l}$ come from either the NM layers (in $\mathrm{Co} / \mathrm{Pd}$ and $\mathrm{Co} / \mathrm{Pt}$ systems) or the atomic Co layers close to the $\mathrm{Co} / \mathrm{NM}$ interfaces (in $\mathrm{Co} / \mathrm{Cu}$ and $\mathrm{Co} / \mathrm{Ag}$ systems). In both cases, these contributions remain almost unchanged upon increasing $N_{\text {Co }}$ since it effectively corresponds to adding $\mathrm{Co}$ atomic layers inside the $\mathrm{Co}$ parts. As a result, according to the formula (4), the sum of the contributions $\alpha_{l}$ from the NM layers and/or from Co atomic layers close to the interfaces gives the leading term, proportional to $1 / N_{\mathrm{Co}}$, in the dependence $\alpha\left(N_{\mathrm{Co}}\right)$. This mechanism is clearly visualized for the $[\mathrm{Co} / \mathrm{Ag}(4 \mathrm{ML})]_{4}$ and $[\mathrm{Co} / \mathrm{Pd}(4 \mathrm{ML})]_{4}$ multilayers with $\Gamma=0.1 \mathrm{eV}$ in Fig. 15 .

Finally, it is found that for a large scattering rate like $\Gamma=0.1 \mathrm{eV}$, the atomic layer contributions are almost constant throughout the Pd layers and have very similar values for different Pd layer thicknesses, from 2 to 8 ML (Fig. 16). A roughly constant distribution of the Gilbert damping is also found in the Pt layers of the Co/Pt systems with $\Gamma=0.1 \mathrm{eV}$ (though $\alpha_{l}$ noticeably decrease in the center of these layers, for $N_{\mathrm{Pt}}=6$ and $8 \mathrm{ML}$ ). Therefore, since the dominant part of the Gilbert damping in the $\mathrm{Co} / \mathrm{Pd}$ and $\mathrm{Co} / \mathrm{Pt}$ multilayers comes from the Pd and Pt layers, respectively, the damping constant for $\Gamma=0.1 \mathrm{eV}$ grows roughly linearly with $N_{\mathrm{NM}}$ as seen in Fig. 10. This elucidates the obtained linear dependence of $\alpha$ on $x=N_{\mathrm{NM}} / N_{\mathrm{Co}}$ with similar slope for different NM thicknesses (see Fig. 9) which was previously observed experimentally $[22,23]$. This property is presently found for $\Gamma=0.1 \mathrm{eV}$ but not for $\Gamma=0.01 \mathrm{eV}$ since in the latter case the layer contributions cease to be roughly constant throughout the NM layers and independent of their thicknesses (see Figs. 16 and 11). For the smaller value of the scattering rate, $\Gamma=0.01 \mathrm{eV}$, the atomic layer damping contributions inside the $\mathrm{Pd}$ and $\mathrm{Pt}$ 

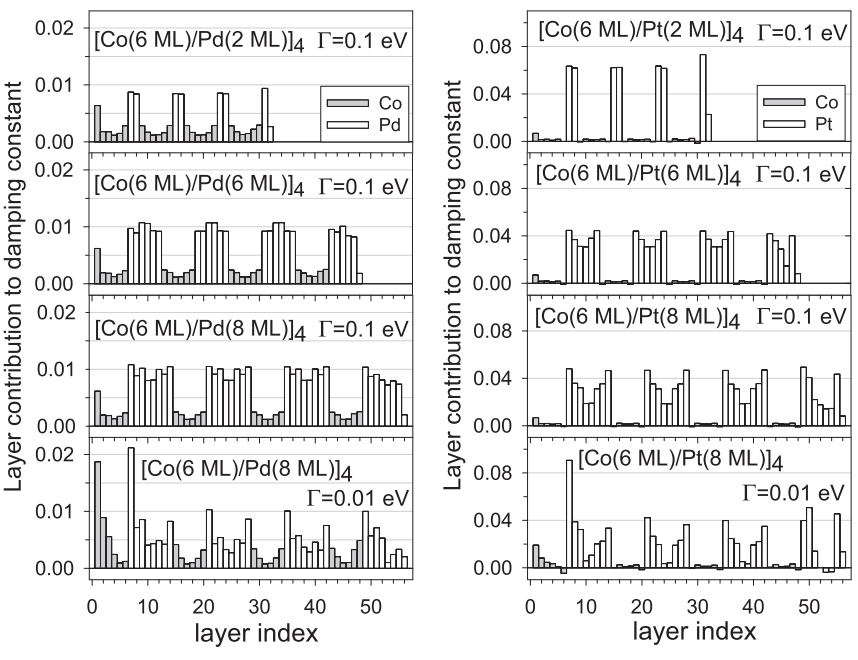

FIG. 16. Layer contributions to the damping constant $\alpha$ in $[\mathrm{Co}(6$ $\left.\mathrm{ML}) / \mathrm{NM}\left(N_{\mathrm{NM}} \mathrm{ML}\right)\right]_{4}$ multilayers $(\mathrm{NM}=\mathrm{Pd}, \mathrm{Pt})$ with $N_{\mathrm{NM}}=2$, 6 , and $8 \mathrm{ML}$ with $\Gamma=0.1 \mathrm{eV}$ as well as for $N_{\mathrm{NM}}=8 \mathrm{ML}$ with $\Gamma=0.01 \mathrm{eV}$. The layer contributions for $N_{\mathrm{NM}}=4 \mathrm{ML}$ and $\Gamma=0.01$ and $0.1 \mathrm{eV}$ are shown in Fig. 11.

layers tend to decline with increasing the distance from the $\mathrm{Co} / \mathrm{NM}$ interfaces, in a similar way as previously found for $\mathrm{Co} / \mathrm{Pd}$ and $\mathrm{Co} / \mathrm{Pt}$ bilayers [57]. Such spatial distribution of the Gilbert damping explains its dependence on the NM layer thickness for $\Gamma=0.01 \mathrm{eV}$ (Fig. 10), with $\alpha$ almost saturated at $N_{\mathrm{NM}}=8 \mathrm{ML}$.

\section{CONCLUSIONS}

Summarizing, the Gilbert damping constant $\alpha$ is calculated quantum mechanically for $[\mathrm{Co} / \mathrm{NM}]_{N}$ magnetic multilayers, including $\mathrm{L}_{0}$ superlattices, by employing the torquecorrelation model within the TB formalism. It is found that $\alpha$ largely depends on the type of the nonmagnetic metallic layers $(\mathrm{NM}=\mathrm{Cu}, \mathrm{Ag}, \mathrm{Au}, \mathrm{Pd}, \mathrm{Pt})$ as well as the parameters that characterize the geometric structure of the multilayers. The origin of these dependences is successfully investigated by making the spatial decomposition of the damping constant into contributions from individual atomic layers.

In particular, it is revealed that the damping constant, in both $\mathrm{L}_{0}[\mathrm{Co}(1 \mathrm{ML}) / \mathrm{NM}(1 \mathrm{ML})]_{N}$ superlattices and multilayers with thicker Co and NM layers, decreases as $c+b / N$ with increasing the stacking number $N$ and saturates after a few repetitions of the $\mathrm{Co} / \mathrm{NM}$ bilayer block for the scattering rates $\Gamma \leqslant 0.02 \mathrm{eV}$, while it is almost independent of $N$ for larger $\Gamma$. Such a dependence on $N$ is shown to arise from the modification of the damping contributions near the external surfaces of the multilayers. The $N$-dependent part of $\alpha$ is found to come from the intraband transitions, which give a negligible contribution to the Gilbert damping at large $\Gamma$.

On the other hand, the introduction of NM layers into a Co film, which leads to the formation of the multilayers with different $N$ but the same total Co thickness, is found to enhance the Gilbert damping for each of the considered nonmagnetic metals. This enhancement results from the strong SO coupling of NM combined with its large DOS at the Fermi level, as in $\mathrm{Pd}$ and $\mathrm{Pt}$, and, to a minor extent, from the modification of the electronic structure in Co layers close to the $\mathrm{Co} / \mathrm{NM}$ interfaces. The prediction of the enhanced damping due to the dominant contributions from the Pd and Pt layers is in a general agreement with the spin pumping theory where the two metals are treated as good spin sinks.

The obtained linear dependence of the damping constant on the inverse of the Co layer thickness in Co/NM multilayers agrees with previous experimental observations $[22,23,44]$ and the very recent theoretical results for inifinite $\mathrm{Co} / \mathrm{Pd}$ and $\mathrm{Co} / \mathrm{Pt}$ superlattices [56]. In addition, the present results found with large scattering rates, close to $\Gamma=0.1 \mathrm{eV}$, reproduce well the experimental finding of the common slope in the linear dependence on the thickness ratio $x=N_{\mathrm{NM}} / N_{\mathrm{Co}}$ in $\mathrm{Co} / \mathrm{NM}$ multilayers with different NM layer thicknesses, reported for both $\mathrm{NM}=\mathrm{Pd}$ and $\mathrm{Pt}[22,23]$. This is shown to be related to the roughly linear dependence of $\alpha$ on the NM layer thickness (in the investigated range of $N_{\mathrm{NM}}$ ) for a large scattering rates which is significantly different from the corresponding dependence for a smaller $\Gamma=0.01 \mathrm{eV}$ considered in our previous work on $\mathrm{Co} / \mathrm{NM}$ bilayers [57]. The dependence on $N_{\mathrm{NM}}$ and $x$ is further explained by analyzing the spatial distribution of the Gilbert damping inside the $\mathrm{Pd}$ and Pt layers.

In conclusion, the present paper gives new insight into the quantum mechanisms of the Gilbert damping in magnetic layered systems, in particular, its dependence on their composition and geometric structure. Apart from leading to deeper understanding of this phenomenon, the obtained results can also be useful for manipulating the magnetic damping in the way desired in various applications.

\section{ACKNOWLEDGMENTS}

We acknowledge the financial support of the Foundation for Polish Science (Poland) within the International Ph.D. Projects Programme, cofinanced from the European Regional Development Fund within Innovative Economy Operational Programme "Grants for innovation". We are also indebted to D. M. Edwards and A. Umerski for inspiring discussions, and to T. Kato for clarification of his experimental results.
[1] S. Veprek, J. Vac. Sci. Technol. A 17, 2401 (1999).

[2] Heterojunctions and Semiconductor Superlattices, edited by G. Allan, G. Bastard, N. Boccara, M. Lannoo, and M. Voos (Springer, Berlin, 1986).

[3] Edited by H. T. Grahn Semiconductor Superlattices: Growth and Electronic Properties (World Scientific, Singapore, 1995).
[4] A. Wacker, Phys. Rep. 357, 1 (2002).

[5] S. S. P. Parkin, R. Bhadra, and K. P. Roche, Phys. Rev. Lett. 66, 2152 (1991).

[6] S. S. P. Parkin, Phys. Rev. Lett. 67, 3598 (1991).

[7] S. S. P. Parkin, in Ultrathin Magnetic Structures II, edited by B. Heinrich and J. A. C. Bland, (Springer-Verlag, Berlin Heidelberg, 1994). 
[8] S. Araki, K. Yasui, and Y. Narumiya, J. Phys. Soc. Jpn. 60, 2827 (1991).

[9] L. Wu, T. Shinjo, and N. Nakayama, J. Magn. Magn. Mater. 125, L14 (1993).

[10] F. Petroff, A. Barthélemy, D. H. Mosca, D. K. Lottis, A. Fert, P. A. Schroeder, W. P. Pratt, Jr., R. Loloee, and S. Lequien, Phys. Rev. B 44, 5355(R) (1991).

[11] Z. Y. Liu, F. Zhang, B. Xu, D. L. Yu, J. L. He, and Y. J. Tian, J. Phys. D: Appl. Phys. 42, 035010 (2009).

[12] L. Li, Y. Lu, Z. Liu, Y. Lv, Y. Zhang, S. Liu, C. Hao, and W. Lv, J. Magn. Magn. Mater. 325, 117 (2013).

[13] M. N. Baibich, J. M. Broto, A. Fert, F. Nguyen Van Dau, F. Petroff, P. Etienne, G. Creuzet, A. Friederich, and J. Chazelas, Phys. Rev. Lett. 61, 2472 (1988).

[14] D. M. Edwards, J. Mathon, and R. B. Muniz, IEEE Trans. Magn. 27, 3548 (1991).

[15] F. J. A. den Broeder, D. Kuiper, H. C. Donkersloot, and W. Hoving, Appl. Phys. A 49, 507 (1989).

[16] C.-J. Lin, G. L. Gorman, C. H. Lee, R. F. C. Farrow, H. V. Notarys, and C. J. Chien, J. Magn. Magn. Mater. 93, 194 (1991).

[17] D. Weller, R. F. C. Farrow, R. F. Marks, G. R. Harp, H. Notarys, and G. Gorman, MRS Symposia Proceedings No. 313 ((Materials Research Society, Pittsburgh, 1993), p. 791.

[18] M. T. Johnson, P. J. H. Bloemen, F. J. A. den Broeder, and J. J. de Vries, Rep. Prog. Phys. 59, 1409 (1996).

[19] K. Yakushiji, T. Saruya, H. Kubota, A. Fukushima, T. Nagahama, S. Yuasa, and K. Ando, Appl. Phys. Lett. 97, 232508 (2010).

[20] S. Emori and G. S. D. Beach, J. Appl. Phys. 110, 033919 (2011).

[21] Z. Liu, R. Brandt, O. Hellwig, S. Florez, T. Thomson, B. Terris, and H. Schmidt, J. Magn. Magn. Mater. 323, 1623 (2011).

[22] T. Kato, Y. Matsumoto, S. Kashima, S. Okamoto, N. Kikuchi, S. Iwata, O. Kitakami, and S. Tsunashima, IEEE Trans. Magn. 48, 3288 (2012)

[23] T. Kato, K. Adachi, Y. Kusanagi, S. Okamoto, N. Kikuchi, O. Kitakami, and S. Iwata, The 38th Annual Conference on Magnetics in Japan, abstract No. 3pB-1, http://www.magnetics.jp/kouenkai/2014/session/nameindex.html\#kk.

[24] H. Shima, K. Oikawa, A. Fujita, K. Fukamichi, K. Ishida, S. Nakamura, and T. Nojima, J. Magn. Magn. Mater. 290-291, 566 (2005).

[25] S. Mizukami, S. Iihama, N. Inami, T. Hiratsuka, G. Kim, H. Naganuma, M. Oogane, and Y. Ando, Appl. Phys. Lett. 98, 052501 (2011).

[26] P. He, X. Ma, J. W. Zhang, H. B. Zhao, G. Lupke, Z. Shi, and S. M. Zhou, Phys. Rev. Lett. 110, 077203 (2013).

[27] X. Ma, L. Ma, P. He, H. B. Zhao, S. M. Zhou, and G. Lüpke, Phys. Rev. B 91, 014438 (2015).

[28] A. D. Kent and D. C. Worledge, Nat. Nanotechnol. 10, 187 (2015).

[29] K. Ando, S. Fujita, J. Ito, S. Yuasa, Y. Suzuki, Y. Nakatani, T. Miyazaki, and H. Yoda, J. Appl. Phys. 115, 172607 (2014).

[30] K. Song and K.-J. Lee, J. Appl. Phys. 118, 053912 (2015).

[31] R. Sbiaa, H. Meng, and S. N. Piramanayagam, Phys. Status Solidi RRL 5, 413 (2011).

[32] C. Burrowes, A. P. Mihai, D. Ravelosona, J.-V. Kim, C. Chappert, L. Vila, A. Marty, Y. Samson, F. Garcia-Sanchez,
L. D. Buda-Prejbeanu, I. Tudosa, E. E. Fullerton, and J.-P. Attané, Nat. Phys. 6, 17 (2010).

[33] S. Bohlens and D. Pfannkuche, Phys. Rev. Lett. 105, 177201 (2010).

[34] J. Chico, C. Etz, L. Bergqvist, O. Eriksson, J. Fransson, A. Delin, and A. Bergman, Phys. Rev. B 90, 014434 (2014).

[35] R. M. Reeve, A. Loescher, M.-A. Mawass, R. Hoffmann-Vogel, and M. Kläui, J. Phys. Condens. Matter 26, 474207 (2014).

[36] T. L. Gilbert, Phys. Rev. 100, 1243 (1955).

[37] T. L. Gilbert, IEEE Trans. Magn. 40, 3443 (2004).

[38] V. Kamberský, Czech. J. Phys., Sec. 26, 1366 (1976).

[39] Y. Tserkovnyak, A. Brataas, and G. E. W. Bauer, Phys. Rev. Lett. 88, 117601 (2002); Phys. Rev. B 66, 224403 (2002); J. Appl. Phys. 93, 7534 (2003).

[40] R. Arias and D. L. Mills, Phys. Rev. B 60, 7395 (1999).

[41] P. Landeros, R. E. Arias, and D. L. Mills, Phys. Rev. B 77, 214405 (2008).

[42] S. J. Yuan, L. Sun, H. Sang, J. Du, and S. M. Zhou, Phys. Rev. B 68, 134443 (2003).

[43] A. Barman, S. Wang, O. Hellwig, A. Berger, E. E. Fullerton, and H. Schmidt, J. Appl. Phys. 101, 09D102 (2007).

[44] S. Pal, B. Rana, O. Hellwig, T. Thomson, and A. Barman, Appl. Phys. Lett. 98, 082501 (2011).

[45] S. Mizukami, X. Zhang, T. Kubota, H. Naganuma, M. Oogane, Y. Ando, and Terunobu Miyazaki, Appl. Phys. Express 4, 013005 (2011).

[46] S. Iihama, M. Khan, H. Naganuma, M. Oogane, T. Miyazaki, S. Mizukami, and Y. Ando, J. Magn. Soc. Jpn. 39, 57 (2015).

[47] S. Mizukami, J. Magn. Soc. Jpn. 39, 1 (2015).

[48] T. Kato, Y. Matsumoto, S. Okamoto, N. Kikuchi, O. Kitakami, N. Nishizawa, S. Tsunashima, and S. Iwata, IEEE Trans. Magn. 47, 3036 (2011).

[49] Y. Nozaki, N. Narita, T. Tanaka, and K. Matsuyama, Appl. Phys. Lett. 95, 082505 (2009).

[50] J.-W. Kim, H.-S. Song, J.-W. Jeong, K.-D. Lee, J.-W. Sohn, T. Shima, and S.-Ch. Shin, Appl. Phys. Lett. 98, 092509 (2011).

[51] M. Haertinger, C. H. Back, S.- H Yang, S. S. P. Parkin, and G. Woltersdorf, J. Phys. D: Appl. Phys. 46, 175001 (2013).

[52] A. Caprile, M. Pasquale, M. Kuepferling, M. Coïsson, T. Y. Lee, and S. H. Lim, IEEE Magn. Lett. 5, 3000304 (2014).

[53] L. Berger, J. Appl. Phys. 90, 4632 (2001).

[54] A. Sakuma, J. Phys. Soc. Jpn. 81, 084701 (2012).

[55] T. Qu and R. H. Victora, Appl. Phys. Lett. 106, 072404 (2015).

[56] T. Qu and R. H. Victora, Phys. Rev. B 93, 224426 (2016).

[57] E. Barati, M. Cinal, D. M. Edwards, and A. Umerski, Phys. Rev. B 90, 014420 (2014).

[58] E. Barati and M. Cinal, Phys. Rev. B 91, 214435 (2015).

[59] D. M. Edwards, J. Phys. Condens. Matter 28, 086004 (2016).

[60] Finite interlayer exchange coupling is reported for $\mathrm{Co} / \mathrm{Ag}$ and $\mathrm{Co} / \mathrm{Au}$ multilayers in Refs. [8,9], respectively, while no such coupling was found for these systems by Parkin [6,7].

[61] K. Gilmore, Y. U. Idzerda, and M. D. Stiles, Phys. Rev. Lett. 99 , 027204 (2007).

[62] E. Barati, M. Cinal, D. M. Edwards, and A. Umerski, EPJ Web Conf. 40, 18003 (2013).

[63] K. Tobari, M. Ohtake, K. Nagano, and M. Futamoto, Jpn. J. Appl. Phys. 50, 073001 (2011).

[64] D. Weller, W. Reim, K. Sprl, and H. Brändle, J. Magn. Magn. Mater. 93, 183 (1991).

[65] S.-K. Kim and S.-C. Shin, J. Appl. Phys. 89, 3055 (2001). 
[66] C.-H. Su, S.-C. Lo, J. van Lierop, K.-W. Lin, and H. Ouyang, J. Appl. Phys. 105, 07C316 (2009).

[67] S. Azzawi, A. Ganguly, M. Tokaç, R. M. Rowan-Robinson, J. Sinha, A. T. Hindmarch, A. Barman, and D. Atkinson, Phys. Rev. B 93, 054402 (2016).

[68] S. Mizukami, Y. Ando, and T. Miyazaki, J. Magn. Magn. Mater. 226-230, 1640 (2001); Jpn. J. Appl. Phys. 40, 580 (2001).

[69] S. M. Bhagat and P. Lubitz, Phys. Rev. B 10, 179 (1974).

[70] T. Kato (private communication).

[71] G. Winer, A. Segal, M. Karpovski, V. Shelukhin, and A. Gerber, J. Appl. Phys. 118, 173901 (2015).
[72] A. Ganguly, S. Azzawi, S. Saha, J. A. King, R. M. RowanRobinson, A. T. Hindmarch, J. Sinha, D. Atkinson, and A. Barman, Sci. Rep. 5, 17596 (2015).

[73] R. H. Victora and J. M. MacLaren, J. Appl. Phys. 69, 5652 (1991).

[74] K. Kyuno, R. Yamamoto, and S. Asano, J. Magn. Magn. Mater. 126, 268 (1993).

[75] D. L. R. Santos, P. Venezuela, R. B. Muniz, and A. T. Costa, Phys. Rev. B 88, 054423 (2013).

[76] A. T. Costa and R. B. Muniz, Phys. Rev. B 92, 014419 (2015). 